J.Math.Phys. (0\%-0355R)

\title{
Lower Spectral Branches of a Spin-Boson Model
}

\author{
Nicolae Angelescu ${ }^{1}$, Robert A. Minlos ${ }^{2}$, Jean Ruiz ${ }^{3}$ \\ and \\ Valentin A. Zagrebnov ${ }^{4}$
}

${ }^{1}$ National Institute of Physics and Nuclear Engineering "H. Hulubei", P. O. Box MG-6, Bucharest, Romania, e-mail: nangel@theory.nipne.ro

${ }^{2}$ Institute for Information Transmissions Problems, Bolshoj Karetny per.19, GSP-4, Moscow 101447, Russia, e-mail: minl@iitp.ru

${ }^{3}$ Centre de Physique Théorique*, Luminy Case 907, Marseille 13288, Cedex 9, France, e-mail: ruiz@cpt.univ-mrs.fr

${ }^{4}$ Université de la Méditerranée and Centre de Physique Théorique* - Luminy, Case 907, Marseille 13288, Cedex 9, France, e-mail: zagrebnov@cpt.univ-mrs.fr

\begin{abstract}
We study the structure of the spectrum of a two-level quantum system weakly coupled to a boson field (spin-boson model). Our analysis allows to avoid the cutoff in the number of bosons, if their spectrum is bounded below by a positive constant.

We show that, for small coupling constant, the lower part of the spectrum of the spin-boson Hamiltonian contains (one or two) isolated eigenvalues and (respectively, one or two) manifolds of atom +1 -boson states indexed by the boson momentum $q$. The dispersion laws and generalized eigenfunctions of the latter are calculated.
\end{abstract}

Keywords: Spin-boson model, two-level system, spectral branch

Mathematical Subject Classification: 81Q10, 47A40, 47A10, 47A55

* UMR CNRS 6207, Universités Aix-Marseille I et II et Sud Toulon-Var, Laboratoire affilié à la FRUMAM 


\section{Contents}

1 Introduction and the main result 2

2 Reduction to a finite number of bosons 5

3 Discrete spectrum $\quad 13$

4 One-boson branches $\quad 17$

5 Conclusion $\quad 27$

6 Appendix $\quad 29$

\section{Introduction and the main result}

The paper is devoted to the study of the low-lying spectrum of the Hamiltonian of the so-called "spin-boson" model. The Hilbert space of the model is

$$
\mathcal{H}=\mathbb{C}^{2} \otimes \mathcal{F}_{s},
$$

where $\mathcal{F}_{s}$ is the symmetric (boson) Fock space (see 4]):

$$
\mathcal{F}_{s}=\mathcal{F}_{s}\left(L^{2}\left(\mathbb{R}^{d}\right)\right)=\bigoplus_{n=0}^{\infty} \mathcal{F}^{(n)},
$$

with $\mathcal{F}^{(0)}=\mathbb{C}, \mathcal{F}^{(n)}=\left(L^{2}\left(\mathbb{R}^{d}\right)\right)_{\text {sym }}^{\otimes n}(n \geq 1)$ the symmetric tensor power, endowed with the scalar product

$$
(\Psi, \Phi)_{\mathcal{F}^{(n)}}= \begin{cases}\psi_{0} \bar{\varphi}_{0}, & \text { if } n=0 \\ (n !)^{-1} \int_{\mathbb{R}^{d n}} \psi_{n}\left(k_{1}, \ldots, k_{n}\right) \overline{\varphi_{n}\left(k_{1}, \ldots, k_{n}\right)} d k_{1} \ldots d k_{n}, & \text { if } n \geq 1 .\end{cases}
$$

The formal Hamiltonian of the "spin-boson" model is defined as an algebraic sum

$$
H=H_{0}+H_{\text {int }},
$$

of the Hamiltonian of non-interacting subsystems of two-level spin and of free boson field:

$$
H_{0}:=\varepsilon \sigma_{3} \otimes \mathbb{I}+\mathbb{I} \otimes \int_{\mathbb{R}^{d}} \omega(q) a^{*}(q) a(q) d q,
$$

and of the Hamiltonian coupled these subsystems:

$$
H_{i n t}:=\alpha \sigma_{1} \otimes \int_{\mathbb{R}^{d}}\left(\lambda(q) a^{*}(q)+\overline{\lambda(q)} a(q)\right) d q .
$$

Here, 
- $\sigma_{3}, \sigma_{1}$, are the Pauli matrices

$$
\sigma_{3}=\left(\begin{array}{cc}
1 & 0 \\
0 & -1
\end{array}\right), \quad \sigma_{1}=\left(\begin{array}{cc}
0 & 1 \\
1 & 0
\end{array}\right)
$$

- $a^{*}(k), a(k)$ are the boson creation and annihilation operators [4]: for $\phi \in L_{2}\left(\mathbb{R}^{d}\right)$, $a^{*}(\phi)=\int a(q)^{*} \overline{\phi(q)} d q, a(\phi)=\int a(q) \phi(q) d q$, which act on the vector

$$
\Psi=\left(\psi_{0}, \psi_{1}\left(k_{1}\right), \ldots, \psi_{n}\left(k_{1}, \ldots, k_{n}\right), \ldots\right) \in \mathcal{F}_{s}
$$

according to the following rules:

$$
\begin{aligned}
& \left(a^{*}(\phi) \Psi\right)_{n}\left(k_{1}, \ldots, k_{n}\right)= \begin{cases}0, & \text { if } n=0, \\
\sum_{i=1}^{n} \psi_{n-1}\left(k_{1}, \ldots, \check{k}_{i}, \ldots k_{n}\right) \overline{\phi\left(k_{i}\right)}, & \text { if } n \geq 1,\end{cases} \\
& (a(\phi) \Psi)_{n}\left(k_{1}, \ldots, k_{n}\right)=\int \psi_{n+1}\left(k_{1}, \ldots, k_{n}, k\right) \phi(k) d k, \quad \text { if } n \geq 0 .
\end{aligned}
$$

- the one-boson spectrum $\omega(k)>0$ (the boson dispersion law) and $\lambda(k)$ (the formfactor) are functions whose properties will be discussed below;

- $\varepsilon>0$ and $\alpha \geq 0$ are real parameters, whereby we suppose the coupling "constant" $\alpha \ll 1$.

The properties we require for $\omega(k), \lambda(k)$ are the following:

(A1) $\omega(\cdot): \mathbb{R}^{d} \rightarrow(0, \infty)$ is a continuously differentiable function, having a unique nondegenerate minimum at the origin $\omega(0)=: \kappa$, and with $\partial \omega(q) \neq 0$ for $q \neq 0$. Moreover, $\lim _{q \rightarrow \infty}|\partial \omega(q)| / \omega(q)=0$

(A2) $\lambda(\cdot): \mathbb{R}^{d} \rightarrow \mathbb{C}$ is a continuously differentiable function, dominated by a bounded positive square integrable function $h: \mathbb{R}^{d} \rightarrow(0,1]$, i.e.

$$
|\lambda(q)| \leq h(q),|\partial \lambda(q)| \leq C h(q)
$$

for some $C>0$;

(A3) on every level set $\Sigma_{x}=\omega^{-1}(x)$ of the function $\omega$, the function $\lambda$ is not identically equal to zero. i.e. for all $\kappa \leq x<\infty,\left.\lambda\right|_{\Sigma_{x}} \neq 0$.

The Hamiltonian $H_{0}$ of the "free" (non-interacting) system has two simple eigenvalues $e_{0}^{0}=-\varepsilon, e_{1}^{0}=\varepsilon$; the corresponding one-dimensional eigenspaces will be denoted $\mathcal{H}_{0,0}^{(i=0,1)} \subset \mathcal{H}$. Besides, there exist two sequences $\mathcal{H}_{0, n}^{(i)}, n=1,2, \ldots(i=0,1)$ of $H_{0^{-}}$ invariant subspaces on $n$-boson states. Under assumption (A1), in each $\mathcal{H}_{0, n}^{(i)}$, the spectrum $\Sigma_{0}$ of $H_{0}$ is continuous and fills the half-infinite intervals $\left[\lambda_{i, n}^{0}, \infty\right)$, where $\lambda_{i, n}^{0}=e_{i}^{0}+n \kappa$ $(i=0,1 ; n=1,2, \ldots)$.

The paper is concerned with the description of the structure of the lower part of the spectrum of the weakly interacting $(0<\alpha \ll 1)$ system. Namely, our main result (see Theorem 5.1) can be formulated as follows: 
(i) Below the continuous spectrum $\Sigma_{0}$, there exist either one, $e_{0}<-\varepsilon$, or two, $e_{0}<-\varepsilon<$ $e_{1}<\varepsilon$, simple eigenvalues of $H$; the corresponding one-dimensional eigenspaces will be denoted $\mathcal{H}_{0}^{(i)}=\left\{\mathbb{C} F_{0}^{(i)}\right\}, i=0,1$.

(ii) In the orthogonal complement $\left[\mathcal{H}_{0}^{(0)} \oplus \mathcal{H}_{0}^{(1)}\right]^{\perp}$ there exist (depending on the number of eigenvalues) either one, or two mutually orthogonal, invariant subspaces $\mathcal{H}_{1}^{(i=0,1)}$, such that the restrictions $\left.H\right|_{\mathcal{H}_{1}^{(i=0,1)}}$ are unitarily equivalent to the operators of multiplication by the functions

$$
\mathcal{E}_{i}(q):=e_{i}+\omega(q), i=0,1,
$$

acting, respectively, in the Hilbert spaces $L_{2}\left(G_{\eta}^{(i=0,1)}\right)$, where the domains $G_{\eta}^{(i=0,1)} \subset$ $\mathbb{R}^{d}$ are defined by

$$
G_{\eta}^{(i)}=\left\{q \in \mathbb{R}^{d}: e_{i}+\omega(q)<\lambda_{i=0, n=2}^{0}-\eta\right\}, i=0,1 .
$$

Here, $0<\eta:=\eta(\alpha)$, where $\eta=\eta(\alpha)$ is small for small $\alpha$. Thereby, the unitaries establishing the equivalence are explicitly constructed.

Remark 1.1 In fact, the two points above exhaust (though this is not explicitly shown in the paper) the spectrum of $H$ in the interval $\left(-\infty, \lambda_{0,2}^{0}-\eta\right)$, meaning that the spectrum of $H$ in the orthogonal complement $\left\{\mathcal{H}_{0}^{(0)} \oplus \mathcal{H}_{0}^{(1)} \oplus \mathcal{H}_{1}^{(0)} \oplus \mathcal{H}_{1}^{(1)}\right\}^{\perp}$ has no point below $\lambda_{0,2}^{0}-\eta$. We did not concentrate here on the problem of completeness, although we think that it is possible within out method. Instead, we focused on the explicit study of the discrete part of the spectrum.

Let us briefly describe our method, by which the subspaces $\mathcal{H}_{n}^{(i)}(n=0,1 ; i=0,1)$ and the spectrum of the Hamiltonian $H$ within them are constructed. It consists in the following: consider the equation

$$
(H-z \mathbb{I}) F=0, F \in \mathcal{H}, z \in \mathbb{R},
$$

which determines the eigenvectors $F$ and eigenvalues $z$. This equation can be written as an infinite system of equations for the components of the vector $F$,

$$
F=\left\{f_{0}(\sigma), f_{1}(\sigma, k), \ldots, f_{n}\left(\sigma, k_{1}, . ., k_{n}\right), \ldots\right\}, \sigma= \pm 1, k_{i} \in \mathbb{R}^{d},
$$

where $f_{n}$ are symmetric functions of the variables $k_{1}, . ., k_{n}$. After eliminating in a special effective way all higher components $f_{n}, n=2,3, \ldots$ from Eq. (1.5)), we are left with an equation for the vector $F_{\leq 1}=\left(f_{0}, f_{1}\right)$ of the form:

$$
A(z) F_{\leq 1}-z F_{\leq 1}=0,
$$

where $\left\{A(\xi), \xi \in\left(-\infty, \lambda_{0,2}^{0}-\eta\right)\right\}$ is a family of generalized Friedrichs operators (see, e.g. 11). For each given $\xi$, the operator $A(\xi)$ has one (or two) eigenvalues $e_{i}(\xi), i=0,1$, which 
can be calculated, e.g. as the zeros of the corresponding Fredholm determinant, while its continuous spectrum is found using scattering theory and coincides with the spectrum of the operators of multiplication by certain functions $e_{i}(q ; \xi)$ in the space $L_{2}\left(\mathbb{R}^{d}\right)$. Finally, the solutions $e_{i}$ of the equations $e_{i}(\xi)=\xi$ define the discrete spectrum of the operator $H$, and the solutions $\xi^{(i)}(q)$ of the equations $e_{i}(q ; \xi)=\xi$, which are shown to be equal to $e_{i}+\omega(q)$, give its continuous spectrum.

The method sketched above has been applied in authors' papers [12], [1], where a model of a quantum particle interacting with a massive scalar Bose field was considered and the lower branch of the spectrum of its Hamiltonian (polaron) has been studied. It has been also used in [13], for the analogous problem in a model of a quantum particle interacting with a massive vector Bose field (similar to the Pauli-Fierz model in electrodynamics).

Remark 1.2 It should be noted that our results concerning the continuous branches of the spectrum of the Hamiltonian 1.1 are already contained in the paper [6], but there all invariant subspaces of $H$ are constructed using the abstract methods of scattering theory, under condition that its eigenvalues and eigenvectors are known.

The essential difference is that we construct the eigenfunctions for discrete spectrum of $H$ as well as generalized eigenfunctions for continuous spectrum (lowest one-boson spectral branches) explicitly.

Besides, some analogous results are contained in the papers [8], [2], [11, [14, and also in [10], 20, however in the latter the Hamiltonian $H$ with a "cutoff in the number of bosons" was considered.

Beside this introduction the paper consists of three sections. In Section 2, the procedure of elimination of the higher components of the vector $F$ from Eq. (1.5) and its reduction to Eq. (1.7) is presented in detail. Thereby we consider directly the general case, where the components $f_{n}, n>n_{0}$ with an arbitrary $n_{0} \geq 0$ are eliminated. In Sections 3, 4. Eq. (1.7) is analyzed for $n_{0}=0$ and $n_{0}=1$ and the invariant subspaces of the operator $H$ indicated above, along with its (discrete and continuous) spectra in them, are constructed.

\section{Reduction to a finite number of bosons}

We shall show here how the spectral problem for $H$ can be reduced, at sufficiently small coupling, to an equivalent problem within the subspace with at most $n$ bosons.

It will be convenient to represent $\mathcal{H}$ as a space of $\mathbb{C}^{2}$-valued functions:

$$
\mathcal{H}=L^{2}\left(\mathcal{C}, \mathbb{C}^{2} ; d \mu\right)
$$

where $\mathcal{C}=\bigcup_{n=0}^{\infty} \mathcal{C}_{n}$ is the set of all finite subsets of $\mathbb{R}^{d}$, thereby $Q \in \mathcal{C}_{n}$ if $|Q|=n$, and $d \mu$ is the so-called Lebesgue-Poisson measure:

$$
d \mu(Q)=(1 /|Q| !) \prod_{q \in Q} d q
$$


Also, for $Q \in \mathcal{C}$, let $\omega(Q)=\sum_{q \in Q} \omega(q)$. To simplify notation, we shall write $Q \backslash q:=$ $Q \backslash\{q\}$ and $Q \cup q:=Q \cup\{q\}$.

The Hamiltonian of the spin-boson model (1.1) writes in this representation

$$
\begin{aligned}
& \left(H_{0} F\right)(Q)=\left[\varepsilon \sigma_{3}+\omega(Q)\right] F(Q) \\
& \left(H_{\text {int }} F\right)(Q)=\alpha \sigma_{1}\left[\sum_{q \in Q} \lambda(q) F(Q \backslash q)+\int \overline{\lambda(k)} F(Q \cup k) d k\right] .
\end{aligned}
$$

Let us consider the orthogonal sum decomposition:

$$
\mathcal{H}=\mathcal{H}_{\leq n} \oplus \mathcal{H}_{>n},
$$

where $\mathcal{H}_{\leq n}=\{F \in \mathcal{H} ; F(Q)=0, \forall Q,|Q|>n\} \sim L^{2}\left(\bigcup_{k \leq n} \mathcal{C}_{k}, \mathbb{C}^{2} ; d \mu\right)$. Accordingly, the Hamiltonian (2.3) has a matrix representation

$$
H=\left(\begin{array}{ll}
A_{n} & C_{n} \\
C_{n}^{*} & B_{n}
\end{array}\right),
$$

where $A_{n}=P_{\mathcal{H}_{\leq n}} H P_{\mathcal{H}_{\leq n}}, B_{n}=P_{\mathcal{H}_{>n}} H P_{\mathcal{H}_{>n}}$ (here, $P_{\mathcal{H}_{>n}}, P_{\mathcal{H}_{>n}}$ denote the orthogonal projections on the corresponding subspaces), and $C_{n}: \mathcal{H}_{>n} \rightarrow \mathcal{H}_{\leq n}$ is given by

$$
\left(C_{n} F\right)(Q)=\delta_{|Q|, n} \alpha \sigma_{1} \int \overline{\lambda(k)} F(Q \cup k) d k,
$$

while $C_{n}^{*}: \mathcal{H}_{\leq n} \rightarrow \mathcal{H}_{>n}$ equals

$$
\left(C_{n}^{*} F\right)(Q)=\delta_{|Q|, n+1} \alpha \sigma_{1} \sum_{k \in Q} F(Q \backslash k) \lambda(k) .
$$

As $\operatorname{Ran}\left(C_{n}\right) \subset \mathcal{H}_{n}, C_{n}^{*}$ can be viewed as an operator : $\mathcal{H}_{n} \rightarrow \mathcal{H}_{>n}$.

The restriction to $\mathcal{H}_{\leq n}$ of the resolvent of the Hamiltonian is obtained by solving for $F_{n} \in \mathcal{H}_{\leq n}, \tilde{F}_{n} \in \mathcal{H}_{>n}$ the system of two equations, where $G \in \mathcal{H}_{\leq n}$ :

$$
\left\{\begin{aligned}
\left(A_{n}-z \mathbb{I}_{\leq n}\right) F_{n} & + & C_{n} \tilde{F}_{n} & =G \\
C_{n}^{*} F_{n} & + & \left(B_{n}-z \mathbb{I}_{>n}\right) \tilde{F}_{n} & =0,
\end{aligned}\right.
$$

where $\mathbb{I}_{\leq n}, \mathbb{I}_{>n}$ are the unit operators in $\mathcal{H}_{\leq n}, \mathcal{H}_{>n}$, respectively.

For $z \in \mathbb{C} \backslash \operatorname{spec}\left(B_{n}\right)$, the second Eq.(2.8) can be solved for $\tilde{F}_{n}$. Upon insertion of the solution into the first Eq.(2.8), one obtains a reduced problem in $\mathcal{H}_{\leq n}$ :

$$
\left(A_{n}-C_{n}\left(B_{n}-z \mathbb{I}_{>n}\right)^{-1} C_{n}^{*}-z\right) F_{n}=G .
$$

If the operator in the l.h.s. is invertible and $F_{n}(z)$ is the solution of Eq.(2.9), then $\left(F_{n}(z), \tilde{F}_{n}(z)\right)$, where

$$
\tilde{F}_{n}(z)=-\left(B_{n}-z \mathbb{I}_{>n}\right)^{-1} C_{n}^{*} F_{n}(z),
$$

is the unique solution of Eq.(2.8) 
Our next task is to obtain a good characterization of the operator

$$
M_{n}(z):=\left(B_{n}-z \mathbb{I}_{>n}\right)^{-1} C_{n}^{*}: \mathcal{H}_{n} \rightarrow \mathcal{H}_{>n} .
$$

Let $\eta>0$, and define

$$
D_{n, \eta}:=\left\{z \in \mathbb{C}: \operatorname{Re} z<\lambda_{0, n+1}^{0}-\eta\right\} .
$$

(We remind that $\lambda_{0, n}^{0}:=-\varepsilon+n \kappa$ is the first threshold of the $n$-boson branch of $H_{0}$.)

Lemma 2.1 There exists $\alpha_{0}=\alpha_{0}(n, \eta)$, such that, for any $\alpha<\alpha_{0}$ and $z \in D_{n, \eta}$, the operator $M_{n}(z), E q$. (2.11), is bounded.

Proof. We represent $B_{n}$ in the form

$$
B_{n}=B_{n}^{0}+V_{n},
$$

where

$$
\begin{gathered}
\left(B_{n}^{0} F\right)(Q)=\left(\varepsilon \sigma_{3}+\omega(Q)\right) F(Q), \\
\left(V_{n} F\right)(Q)=\alpha \sigma_{1}\left[\sum_{k \in Q} \lambda(k) F(Q \backslash k)+\int \overline{\lambda(k)} F(Q \cup k) d k\right], F \in \mathcal{H}_{>n} .
\end{gathered}
$$

Hence, $M_{n}(z)$ is (formally) represented as

$$
M_{n}(z)=\left(\mathbb{I}_{>n}+\left(B_{n}^{0}-z \mathbb{I}_{>n}\right)^{-1} V_{n}\right)^{-1}\left(B_{n}^{0}-z \mathbb{I}_{>n}\right)^{-1} C_{n}^{*} .
$$

The assertion of Lemma 2.1 follows from the representation (2.13), if we prove that:

1. $\left\|\left(B_{n}^{0}-z \mathbb{I}_{>n}\right)^{-1} V_{n}\right\|_{\mathcal{H}>n}<1$ for $z \in D_{n, \eta}$ and $\alpha$ sufficiently small;

2. $\left.\| B_{n}^{0}-z \mathbb{I}_{>n}\right)^{-1} C_{n}^{*} \|_{\mathcal{H}>n}<\infty$.

To prove 1 we split $\left(B_{n}^{0}-z \mathbb{I}_{>n}\right)^{-1} V_{n}$ into the sum of two terms:

$$
\begin{aligned}
& \left(S_{1}(z) F\right)(Q)= \begin{cases}\left(\varepsilon \sigma_{3}+\omega(Q)-z\right)^{-1} \alpha \sigma_{1} \sum_{q \in Q} F(Q \backslash q) \lambda(q), & \text { if }|Q|>n+1 \\
0, & \text { if }|Q|=n+1\end{cases} \\
& \left(S_{2}(z) F\right)(Q)=\left(\varepsilon \sigma_{3}+\omega(Q)-z\right)^{-1} \alpha \sigma_{1} \int \overline{\lambda(k)} F(Q \cup k) d k
\end{aligned}
$$

and estimate separately their norms.

Let $F_{l} \in L_{2}\left(\mathbb{R}^{d l}\right)$ be the components of $F$. We have, for $l \geq n+2$,

$$
\left\|\left(S_{1}(z) F\right)_{l}\right\|_{L_{2}\left(\mathbb{R}^{d l}\right)}<\frac{l \alpha\|\lambda\|_{L_{2}\left(\mathbb{R}^{d}\right)}}{(l-n-1) \kappa+\eta}\left\|F_{l-1}\right\|_{L_{2}\left(\mathbb{R}^{d(l-1)}\right)} .
$$


Hence,

$$
\begin{aligned}
\left\|S_{1}(z) F\right\|_{\mathcal{H}_{>n}}^{2} & =\sum_{l \geq n+1} \frac{1}{l !}\left\|\left(S_{1}(z) F\right)_{l}\right\|_{L_{2}\left(\mathbb{R}^{d l}\right)}^{2} \\
& \leq \alpha^{2}\|\lambda\|_{L_{2}\left(\mathbb{R}^{d}\right)}^{2} \sum_{l \geq n+2} \frac{l}{((l-n-1) \kappa+\eta)^{2}} \frac{\left\|F_{l-1}\right\|_{L_{2}\left(\mathbb{R}^{d(l-1)}\right)}^{2}}{(l-1) !} \\
& \leq \alpha^{2}\|\lambda\|_{L_{2}\left(\mathbb{R}^{d}\right)}^{2} \max _{l \geq n+2} \frac{l}{((l-n-1) \kappa+\eta)^{2}}\|F\|_{\mathcal{H}_{>n}}^{2}=\alpha^{2} \frac{(n+2)\|\lambda\|_{L_{2}\left(\mathbb{R}^{d}\right)}^{2}}{(\kappa+\eta)^{2}}\|F\|_{\mathcal{H}_{>n}}^{2} .
\end{aligned}
$$

A similar calculation gives the following estimate of the second term:

$$
\left\|S_{2}(z) F\right\|_{\mathcal{H}>n}^{2} \leq \alpha^{2} \frac{(n+1)\|\lambda\|_{L_{2}\left(\mathbb{R}^{d}\right)}^{2}}{\eta^{2}}\|F\|_{\mathcal{H}>n}^{2} .
$$

As a consequence, the inequality in 1 holds for $z \in D_{n, \eta}$, if

$$
\alpha<\frac{1}{2\|\lambda\|_{L_{2}\left(\mathbb{R}^{d}\right)}} \min \left\{\frac{\kappa+\eta}{\sqrt{n+2}}, \frac{\eta}{\sqrt{n+1}}\right\}
$$

Point 2 can be proved similarly.

We shall show that, for $z \in D_{n, \eta}$ and for $\alpha$ sufficiently small, $M_{n}(z)$, Eq.(2.11) has a particular representation, which we now define. Let $\mathcal{M}_{2}$ be the space of square $2 \times 2$ complex matrices with some norm $|\cdot|$ (e.g. $\left.|n|=\frac{1}{2} \max _{i, j}\left|n_{i, j}\right|\right)$, and $h: \mathbb{R}^{d} \rightarrow(0,1]$ be the continuous square-integrable function appearing in assumption (A2).

Definition 2.2 An operator $M_{n}: \mathcal{H}_{n} \rightarrow \mathcal{H}_{>n}$ is said to have a h-regular representation in terms of coefficient functions, if there exist continuously differentiable $\mathcal{M}_{2}$-valued functions

$$
\mu_{n}^{(m)}(\cdot ; \cdot ; \cdot): \mathcal{C} \times \mathcal{C}_{n-m} \times\left(\mathbb{R}^{d}\right)^{m} \rightarrow \mathcal{M}_{2}, \quad m=0,1, \ldots, n,
$$

where $\mu_{n}^{(m)}\left(Q_{1} ; \cdot ; \cdot\right)=0$ for $\left|Q_{1}\right| \leq m$, and $\mu_{n}^{(m)}\left(Q_{1} ; Q_{2} ; \cdot\right)=0$ for $Q_{1} \cap Q_{2} \neq \emptyset$, satisfying, for some $M>0$, the estimation

$$
\sup _{Q_{2} \in \mathcal{C}_{n-m}} \max _{|\alpha| \leq 1}\left|\partial^{\alpha} \mu_{n}^{(m)}\left(Q_{1} ; Q_{2} ; k_{1}, \ldots, k_{m}\right)\right|<M \prod_{q \in Q_{1}} h(q) \prod_{i=1}^{m} h\left(k_{i}\right),
$$

such that, for $f \in \mathcal{H}_{n}$ and $|Q|>n$,

$$
\begin{array}{r}
{\left[M_{n} f\right](Q)=\sum_{m=0}^{n} \sum_{\hat{Q} \subset Q,|\hat{Q}|=n-m} \int \mu_{n}^{(m)}\left(Q \backslash \hat{Q} ; \hat{Q} ; k_{1}, \ldots, k_{m}\right)} \\
\times f\left(\hat{Q} \cup\left\{k_{1}, \ldots, k_{m}\right\}\right) d k_{1} \ldots d k_{m} .
\end{array}
$$


Here and below, we use the notation: for a multiindex $\alpha=\left\{\alpha_{k}^{i} ; k=1, \ldots, n, i=1, \ldots, d\right\}$ and $Q=\left\{q_{1}, \ldots, q_{n}\right\} \in \mathcal{C}_{n}$,

$$
\begin{aligned}
\left(\partial^{\alpha} f\right)(Q) & =\prod_{i, k} \frac{\partial^{\alpha_{k}^{i}}}{\partial\left(q_{k}^{i}\right)^{\alpha_{k}^{i}}} f(Q), \\
|\alpha| & =\sum_{i, k} \alpha_{k}^{i} .
\end{aligned}
$$

In Eq. (2.15), the sum over $\hat{Q}$ is a symmetrization, so that the l.h.s. depends only on the set $Q$. As $f$ is permutation symmetric, it is not necessary to impose the symmetry of $\mu_{n}^{(m)}$ with respect to the $k$ 's. The estimation (2.14) means that the function $\mu_{n}^{(m)}$ and its gradient are bounded uniformly, in particular $M$ is independent of $\left|Q_{1}\right|$. The set of all coefficient functions for a given $h$ and $n$ is a Banach space $\mathcal{B}_{n}$ with the norm $\left\|\mu_{n}\right\|=\inf M$, where the infimum is over all $M$ for which Eq.(2.14) holds.

In order to show that $M_{n}(z)$ has a regular representation for all $z \in D_{n, \eta}$ and determine the corresponding functions $\mu_{n}^{(m)}(z)$, we shall use the identity

$$
\left[1+\left(B_{n}^{(0)}-z \mathbb{I}_{>n}\right)^{-1} V_{n}\right] M_{n}(z)=\left(B_{n}^{(0)}-z \mathbb{I}_{>n}\right)^{-1} C_{n}^{*} .
$$

Suppose an operator $M_{n}: \mathcal{H}_{n} \rightarrow \mathcal{H}_{>n}$ is defined as in Eq.(2.15) by the coefficient functions $\mu_{n}=\left\{\mu_{n}^{(m)}\left(Q_{1} ; Q_{2} ; k_{1}, \ldots, k_{m}\right)\right\}$, where $\left|Q_{2}\right|=n-m$. Then, one can easily see that $\left(B_{n}^{(0)}-z \mathbb{I}_{>n}\right)^{-1} V_{n} M_{n}$ is likewise defined by a sequence of coefficient functions, $\left\{\left[\Gamma(z) \mu_{n}\right]^{(m)}, m=0, \ldots, n\right\}$, with

$$
\begin{aligned}
{\left[\Gamma(z) \mu_{n}\right]^{(m)}\left(Q_{1} ; Q_{2} ; k_{1}, \ldots, k_{m}\right) } & =\alpha\left(\varepsilon \sigma_{3}+\omega\left(Q_{1} \cup Q_{2}\right)-z\right)^{-1} \sigma_{1} \\
& \times\left\{\sum_{q \in Q_{1}} \lambda(q) \mu_{n}^{(m)}\left(Q_{1} \backslash q ; Q_{2} ; k_{1}, \ldots, k_{m}\right)\right. \\
& +\int \bar{\lambda}\left(q^{\prime}\right) \mu_{n}^{(m)}\left(Q_{1} \cup q^{\prime} ; Q_{2} ; k_{1}, \ldots, k_{m}\right) d q^{\prime} \\
& \left.+\bar{\lambda}\left(k_{m}\right) \mu_{n}^{(m-1)}\left(Q_{1} ; Q_{2} \cup k_{m} ; k_{1}, \ldots, k_{m-1}\right)\right\},
\end{aligned}
$$

where the last term does not appear if $m=0$. Let us also note that the r.h.s. of Eq.(2.16) allows the representation Eq.(2.15) with the coefficient functions $\hat{\mu}_{n}(z)$ :

$$
\left[\hat{\mu}_{n}(z)\right]^{(m)}\left(Q_{1}, Q_{2} ; k_{1}, . ., k_{m}\right)=\delta_{m, 0} \delta_{\left|Q_{1}\right|, 1} \alpha\left[\varepsilon \sigma_{3}+\omega\left(Q_{2} \cup q_{1}\right)-z\right]^{-1} \sigma_{1} \lambda\left(q_{1}\right),
$$

where we put $Q_{1}=\left\{q_{1}\right\}$. Therefore, Eq.(2.16) can be written as a fixed-point equation for the coefficients $\mu_{n}=\mu_{n}(z)$ :

$$
\mu_{n}+\Gamma(z) \mu_{n}=\hat{\mu}_{n}(z) .
$$

Proposition 2.3 For any $\eta>0$ and $n \geq 0$, there exists $\alpha_{0}(\eta, n)>0$, such that for any $\alpha<\alpha_{0}(\eta, n)$, and for all $z \in D_{n, \eta}$, Eq. (2.19) has a unique solution $\mu_{n}(z) \in \mathcal{B}_{n}$, which is $\mathcal{B}_{n}$-valued analytic in $D_{n, \eta}$ and $\left\|\mu_{n}(z)\right\| \leq K \alpha / \eta$ for some constant $K$. Moreover,

$$
\mu_{n}^{(m)}\left(z ; Q_{1} ; Q_{2} ; k_{1}, \ldots, k_{m}\right)=\mu_{m}^{(m)}\left(z-\omega\left(Q_{2}\right) ; Q_{1} ; \emptyset ; k_{1}, \ldots, k_{m}\right)
$$


Proof. As both sides of Eq. (2.17) defining $\Gamma(z)$ contain the same $Q_{2}$ and the same $m$ tuple $\left(k_{1}, \ldots, k_{m}\right)$, it will be convenient to treat these variables as parameters, define (for $\left.\left|Q_{2}\right|=n-m\right)$ the functions of $Q_{1}$ :

$$
\nu_{Q_{2},\left(k_{1}, \ldots, k_{m}\right)}^{(m)}\left(Q_{1}\right):=\mu_{n}^{(m)}\left(Q_{1} ; Q_{2} ; k_{1}, \ldots, k_{m}\right)
$$

and remark that

$$
\begin{aligned}
& {\left[\Gamma(z) \mu_{n}\right]^{(m)}\left(Q_{1} ; Q_{2} ; k_{1}, \ldots, k_{m}\right)=\left[\Delta_{m}\left(z-\omega\left(Q_{2}\right)\right) \nu_{Q_{2},\left(k_{1}, \ldots, k_{m}\right)}^{(m)}\right]\left(Q_{1}\right)} \\
& +\alpha\left[\varepsilon \sigma_{3}+\omega\left(Q_{1}\right)-\left(z-\omega\left(Q_{2}\right)\right)\right]^{-1} \cdot \sigma_{1} \lambda\left(k_{m}\right) \nu_{Q_{2} \cup k_{m},\left(k_{1}, \ldots, k_{m-1}\right)}^{(m-1)}\left(Q_{1}\right),
\end{aligned}
$$

where we used that $\omega\left(Q_{1} \cup Q_{2}\right)=\omega\left(Q_{1}\right)+\omega\left(Q_{2}\right)$ for $Q_{1} \cap Q_{2}=\emptyset$. The operator $\Delta_{m}(z), z \in D_{m, \eta}$ acts on the functions $\nu(Q)$ (such that $\nu(Q)=0$ for $|Q| \leq m$ ) according to the formula

$$
\begin{aligned}
\left(\Delta_{m}(z) \nu\right)(Q)= & \chi_{>m}(Q) \alpha\left(\varepsilon \sigma_{3}+\omega(Q)-z\right)^{-1} \\
& \times \sigma_{1}\left\{\sum_{q \in Q} \lambda(q) \nu(Q \backslash q)+\int \overline{\lambda(k)} \nu(Q \cup k) d k\right\},
\end{aligned}
$$

where $\chi_{>m}(Q)$ is the indicator of the set $\mathcal{C}_{>m}$.

Let us consider the Banach space $\tilde{\mathcal{B}}$ of all continuously differentiable functions $\{\nu$ : $\left.\mathcal{C} \rightarrow \mathcal{M}_{2}\right\}$, for which

$$
\|\nu\|=\sup _{Q \in \mathcal{C}} \frac{\max _{|\alpha| \leq 1}\left|\partial^{\alpha} \nu(Q)\right|}{\prod_{q \in Q} h(q)}<\infty,
$$

where $|\cdot|$ denotes the norm in $\mathcal{M}_{2}$. Also, let $\tilde{\mathcal{B}}_{>m} \subset \tilde{\mathcal{B}}$ be the subspace of functions $\nu$ which vanish on $\mathcal{C}_{\leq m}$, i.e. $\nu(Q)=0, \forall Q,|Q| \leq m$.

Lemma 2.4 For every $\eta>0$ and $m \geq 0$, there exists $\hat{\alpha}_{0}(\eta, m)>0$, such that for any $\alpha<\hat{\alpha}_{0}(\eta, m), \Delta_{m}(z)$ is a bounded operator in $\tilde{\mathcal{B}}_{>m}$, norm-analytic of $z$ in $D_{m, \eta}$, and

$$
\sup _{z \in D_{m, \eta}}\left\|\Delta_{m}(z)\right\| \leq 1 / 2
$$

Also, the function

$$
\hat{\nu}\left(z-\omega\left(Q_{2}\right) ; \cdot\right):=\hat{\mu}_{n}^{(0)}\left(z ; \cdot ; Q_{2} ; \emptyset\right)=\hat{\mu}_{0}^{(0)}\left(z-\omega\left(Q_{2}\right) ; \cdot ; \emptyset ; \emptyset\right)
$$

is a $\tilde{\mathcal{B}}_{>0}$-valued analytic function of $z \in D_{n, \eta}\left(n=\left|Q_{2}\right|\right)$ and $\left\|\hat{\nu}\left(z-\omega\left(Q_{2}\right)\right)\right\| \leq C \alpha / \eta$ in $D_{n, \eta}$ for a certain constant $C$.

The proof of this lemma is similar to that of Lemma 2.1 
Remark 2.5 We shall consider also the Banach space $\tilde{\tilde{\mathcal{B}}}$ consisting of continuously differentiable functions $\nu: \mathcal{C} \rightarrow \mathbb{C}^{2}$ with the norm $\|\nu\|$ given by Eq. (2.24), in which now $|\cdot|$ means the usual norm on $\mathbb{C}^{2}$. Its subspaces $\tilde{\tilde{\mathcal{B}}}_{n}, \tilde{\tilde{\mathcal{B}}}_{\leq n}, \tilde{\tilde{\mathcal{B}}}_{>n}$ are introduced as above. It follows from Proposition 2.3 and the representation (2.15) that, for all $z \in D_{n, \eta}, M_{n}(z)$ applies the space $\tilde{\tilde{\mathcal{B}}}_{n}^{\prime}$, the dual space of $\tilde{\tilde{\mathcal{B}}}_{n}$, into $\tilde{\tilde{\mathcal{B}}}_{>n}^{\prime} \subset \mathcal{H}_{>n}$ and is bounded with respect to the norm of $\tilde{\tilde{\mathcal{B}}}^{\prime}$. Besides, assumptions (A1) and (A2) imply that the operator $H$ can be extended to an unbounded operator acting in $\tilde{\tilde{\mathcal{B}}^{\prime}}$ (denoted also $H$ ) on a domain including all finite (with respect to the spatial variables, as well as to the number of variables) elements of $\tilde{\tilde{\mathcal{B}}^{\prime}}$ :

$$
\left\{\phi \in \tilde{\tilde{\mathcal{B}}^{\prime}}: \phi(Q)=0, \text { if } \exists R, \exists N, \operatorname{dist}(0, Q)>R \text { or }|Q|>N\right\}
$$

In this way, as follows from Eq. (2.22),$\nu_{Q_{2}, \emptyset}^{(0)}(\cdot)$ satisfies the equation (2.19) for $m=0$, which writes as

$$
\nu(\cdot)+\Delta_{0}\left(z-\omega\left(Q_{2}\right)\right) \nu(\cdot)=\hat{\nu}\left(z-\omega\left(Q_{2}\right) ; \cdot\right) .
$$

If $z \in D_{n, \eta}$, the difference $z-\omega\left(Q_{2}\right)$ belongs to $D_{0, \eta}$, therefore, according to Lemma 2.4, for $\alpha<\alpha_{0}(\eta, 0)$, the equation has one solution

$$
\nu_{Q_{2}, \emptyset}^{(0)}(z ; \cdot)=\nu_{\emptyset, \emptyset}^{(0)}\left(z-\omega\left(Q_{2}\right) ; \cdot\right) \in \tilde{\mathcal{B}}_{>0},
$$

where the equality comes from the fact that both sides obey the same equation (2.27), therefore both equal its unique solution, $\left(\mathbb{I}+\Delta_{0}\left(z-\omega\left(Q_{2}\right)\right)^{-1} \hat{\nu}\left(z-\omega\left(Q_{2}\right)\right)\right.$. Obviously, the solution is analytic of $z \in D_{n, \eta}$ and (by the smoothness of $\omega$ ) continuously differentiable of $Q_{2}=\left\{q_{1}, \ldots, q_{n}\right\}$. For its norm we have, by Lemma 2.4 , the estimate:

$$
\sup _{z \in D_{n, \eta}, Q_{2} \in \mathcal{C}_{n}}\left\|\nu_{Q_{2}, \emptyset}^{(0)}(z)\right\| \leq \sup _{z \in D_{0, \eta}}\left\|\left(\mathbb{I}+\Delta_{0}(z)\right)^{-1}\right\|\|\hat{\nu}(z)\|<2 C \alpha / \eta .
$$

We consider next the case $m>0, m \leq n$. Eq. (2.19) satisfied by $\nu_{Q_{2},\left(k_{1}, . ., k_{m}\right)}^{(m)}(\cdot)$ writes

$$
\begin{aligned}
& \nu(\cdot)+\Delta_{m}\left(z-\omega\left(Q_{2}\right)\right) \nu(\cdot) \\
& =-\alpha\left[\varepsilon \sigma_{3}+\omega(\cdot)-\left(z-\omega\left(Q_{2}\right)\right)\right]^{-1} \sigma_{1} \lambda\left(k_{m}\right) \nu_{Q_{2} \cup k_{m},\left(k_{1}, \ldots, k_{m-1}\right)}^{(m-1)}(z ; \cdot),
\end{aligned}
$$

and, for $\alpha<\alpha_{0}(\eta, m)$, allows to determine inductively $\nu_{Q_{2},\left(k_{1}, \ldots, k_{m}\right)}^{(m)}(z ; \cdot)$ in terms of the solution $\nu_{Q_{2} \cup k_{m},\left(k_{1}, \ldots, k_{m-1}\right)}^{(m-1)}(z ; \cdot)$ of the $(m-1)$ th equation. Suppose that $\nu_{Q_{2}^{\prime},\left(k_{1}, \ldots, k_{m-1}\right)}^{(m-1)}(z)(\cdot)$ has been shown to fulfill:

1. For $z \in D_{\left|Q_{2}^{\prime}\right|+m-1, \eta}$,

$$
\nu_{Q_{2}^{\prime},\left(k_{1}, \ldots, k_{m-1}\right)}^{(m-1)}(z)=\nu_{\emptyset,\left(k_{1}, \ldots, k_{m-1}\right)}^{(m-1)}\left(z-\omega\left(Q_{2}^{\prime}\right)\right) \in \mathcal{B}_{>m-1},
$$

and

$$
\left\|\nu_{\emptyset,\left(k_{1}, \ldots, k_{m-1}\right)}^{(m-1)}(z)\right\|_{\tilde{\mathcal{B}}_{>m-1}}<C 2^{m}(\alpha / \eta)^{m} \prod_{i=1}^{m-1} h\left(k_{i}\right)
$$


2. $\nu_{\emptyset,\left(k_{1}, \ldots, k_{m-1}\right)}^{(m-1)}(z)$ is a $\tilde{\mathcal{B}}_{>m-1}$-analytic function of $z \in D_{m-1, \eta}$, differentiable of $k_{1}, \ldots, k_{m-1}$ and

$$
\sup _{z \in D_{m-1, \eta}} \max _{1 \leq i \leq m-1}\left|\partial_{k_{i}} \nu_{\emptyset,\left(k_{1}, \ldots, k_{m-1}\right)}^{(m-1)}(z ; Q)\right| \leq C^{\prime} 2^{m}(\alpha / \eta)^{m} \prod_{i=1}^{m-1} h\left(k_{i}\right) \prod_{q \in Q} h(q),
$$

where the constants $C, C^{\prime}$ do not depend on $m, k_{1}, \ldots, k_{m-1}, Q$.

Remark that, by Eqs. (2.28) and (2.29), $\nu_{Q_{2}, \emptyset}^{(0)}(z)$ fulfills both conditions.

Using Lemma 2.4 we find that Eq. (2.30) has, for $\alpha<\alpha_{0}(\eta, m)$ and $z \in D_{\left|Q_{2}\right|+m}$, one solution

$$
\begin{aligned}
& \nu_{Q_{2},\left(k_{1}, \ldots, k_{m}\right)}^{(m)}(z ; \cdot) \\
& =\left(\mathbb{I}+\Delta_{m}\left(z-\omega\left(Q_{2}\right)\right)\right)^{-1} \alpha\left[\varepsilon \sigma_{3}+\omega(\cdot)-\left(z-\omega\left(Q_{2}\right)\right)\right]^{-1} \sigma_{1} \lambda\left(k_{m}\right) \nu_{Q_{2} \cup k_{m},\left(k_{1}, \ldots, k_{m-1}\right)}^{(m-1)}(z ; \cdot)
\end{aligned}
$$

which has the analogous properties. This proves the existence of the coefficient functions $\mu_{n}^{(m)}\left(z ; Q_{1} ; Q_{2} ; k_{1}, \ldots, k_{m}\right)$ and their estimates (2.14).

The equality (2.31) in the case $m>0$ follows again by induction with respect to $m$ :

Taking $Q_{2}=\emptyset$ in Eq. (2.34), we have:

$$
\begin{aligned}
& \nu_{\emptyset,\left(k_{1}, \ldots, k_{m}\right)}^{(m)}(z) \\
& \left.=\left(\mathbb{I}+\Delta_{m}(z)\right)^{-1} \alpha\left[\varepsilon \sigma_{3}+\omega(\cdot)-z\right)\right]^{-1} \sigma_{1} \lambda\left(k_{m}\right) \nu_{\left\{k_{m}\right\},\left(k_{1}, \ldots, k_{m-1}\right)}^{(m-1)}(z ; \cdot)
\end{aligned}
$$

Suppose that (2.31) holds true; then,

$\nu_{Q_{2} \cup k_{m},\left(k_{1}, \ldots, k_{m-1}\right)}^{(m-1)}(z ; \cdot)=\nu_{\left\{k_{m}\right\},\left(k_{1}, \ldots, k_{m-1}\right)}^{(m-1)}\left(z-\omega\left(Q_{2}\right) ; \cdot\right)=\nu_{\emptyset,\left(k_{1}, \ldots, k_{m-1}\right)}^{(m-1)}\left(z-\omega\left(Q_{2}\right)-\omega\left(k_{m}\right) ; \cdot\right)$,

wherefrom it follows that the r.h.s. of Eq. (2.35) written for $z \mapsto z-\omega\left(Q_{2}\right)$ coincides with the r.h.s. of Eq. (2.34). This proves (2.31) for $m$, hence the equality (2.201).

The proposition 2.3 is proved.

The following corollary collects the information on the structure of the operator $C_{n}\left(B_{n}-z \mathbb{I}_{>n}\right)^{-1} C_{n}^{*}$ acting in $\mathcal{H}_{n}$ implied by the regular representation of $M_{n}(z)$.

Corollary 2.6 The following representation holds: for $\alpha<\alpha_{0}(\eta, n)$, and $f \in \mathcal{H}_{n}, Q \in$ $\mathcal{C}_{n}$

$$
\begin{aligned}
& {\left[C_{n}\left(B_{n}-z \mathbb{I}_{>n}\right)^{-1} C_{n}^{*} f\right](Q)=m_{n}(z ; Q) f(Q)+} \\
& \sum_{m=1}^{n} \sum_{Q_{1} \subset Q ;\left|Q_{1}\right|=m} \int \hat{D}_{n, m}\left(z ; Q_{1} ; Q \backslash Q_{1} ; k_{1}, \ldots, k_{m}\right) f\left(\left(Q \backslash Q_{1}\right) \cup\left\{k_{1}, \ldots, k_{m}\right\}\right) d k_{1} \ldots d k_{m},
\end{aligned}
$$

where

1. $m_{n}(z ; Q)=m_{0}(z-\omega(Q) ; \emptyset)$ is analytic of $z \in D_{n, \eta}$, and

$$
\left|m_{n}(z ; Q)\right| \leq 2 C^{\prime}\left(\alpha^{2} / \eta\right)\|\lambda\|_{L_{2}\left(\mathbb{R}^{d}\right)}^{2} ;
$$

Hence, $m_{n}(z ; Q)$ is continuously differentiable of $Q \in \mathcal{C}_{n}$, as well. 
2. $\hat{D}_{n, m}\left(z ; Q_{1} ; Q_{2} ; k_{1}, \ldots, k_{m}\right)=\hat{D}_{m, m}\left(z-\omega\left(Q_{2}\right) ; Q_{1} ; \emptyset ; k_{1}, \ldots, k_{m}\right)$ is analytic of $z \in$ $D_{n, \eta}$, continuously differentiable of $\left\{Q_{1} ; Q_{2} ; k_{1}, \ldots, k_{m}\right\} \in \mathcal{C}_{m} \times \mathcal{C}_{n-m} \times\left(\mathbb{R}^{d}\right)^{m}$, and

$$
\max _{|\alpha| \leq 1}\left|\partial^{\alpha} \hat{D}_{n, m}\left(z ; Q_{1} ; Q_{2} ; k_{1}, \ldots, k_{m}\right)\right| \leq C^{\prime}(2 \alpha / \eta)^{m+1} \prod_{q \in Q_{1}} h(q) \prod_{i=0}^{m} h\left(k_{i}\right),
$$

where $C^{\prime}$ is a constant.

Proof. The assertions follow by applying the representation (2.15), the identity Eq.(2.20) and the estimates (2.32), (2.33) in the formulae:

$$
\begin{aligned}
m_{n}(z ; Q) & =\alpha \sigma_{1} \int \overline{\lambda\left(q^{\prime}\right)} \mu_{n}^{(0)}\left(z ; q^{\prime} ; Q ; \emptyset\right) d q^{\prime}, \\
\hat{D}_{n, m}\left(z ; Q_{1} ; Q_{2} ; k_{1}, \ldots, k_{m}\right) & =\alpha \sigma_{1} \int \overline{\lambda\left(q^{\prime}\right)} \mu_{n}^{(m)}\left(z ; Q_{1} \cup q^{\prime} ; Q_{2} ; k_{1}, \ldots, k_{m}\right) d q^{\prime} \\
& +\alpha \sigma_{1} \overline{\lambda\left(k_{m}\right)} \mu_{n}^{(m-1)}\left(z ; Q_{1} ; Q_{2} \cup k_{m} ; k_{1}, \ldots, k_{m-1}\right) .
\end{aligned}
$$

The $\mathcal{M}_{2}$-valued kernels $\hat{D}\left(Q_{1} ; Q_{2} ; k_{1}, \ldots, k_{m}\right)$, which are continuously differentiable on $\mathcal{C}_{m} \times\left(\mathbb{R}^{d}\right)^{m}$ and dominated by $h$ as in Eq.(2.37), form a Banach space $\mathcal{K}_{m}$ with the norm

$$
\|\hat{D}\|=\sup _{Q_{1}, Q_{2}, k_{1}, \ldots, k_{m}} \max _{|\alpha| \leq 1}\left|\partial^{\alpha} \hat{D}\left(Q_{1} ; Q_{2} ; k_{1}, \ldots, k_{m}\right)\right| / \prod_{q \in Q_{1}} h(q) \prod_{i=0}^{m} h\left(k_{i}\right),
$$

where $|\cdot|$ is the norm in $\mathcal{M}_{2}$.

\section{Discrete spectrum}

We consider here, as an example of the general analysis, the cases $n=0,1$. This allows the construction of the eigenvectors and of part of the one-boson branch. In this section we consider the discrete part of the spectrum.

I. For $n=0$, the equation (2.9) becomes an equation in $\mathbb{C}^{2}$ :

$$
\left(\varepsilon \sigma_{3}-m_{0}(z ; \emptyset)-z\right) f=g,
$$

where the matrix $m_{0}(z ; \emptyset)$ is analytic in $D_{0, \eta}$ and $\left|m_{0}(z ; \emptyset)\right| \leq 2 C^{\prime}\|\lambda\|^{2} \alpha^{2} / \eta$. Eq.(3.1) has a unique solution unless $z$ is a (real) zero of the determinant of the matrix in the l.h.s., i.e., denoting $m(z):=m_{0}(z ; \emptyset)$,

$$
\operatorname{det}\left(\varepsilon \sigma_{3}-m(z)-z\right)=0 .
$$


This equation can be brought to the form

$$
1+\frac{m(z)_{11}+(1 / 2 \varepsilon) \operatorname{det}(m(z))}{z-\varepsilon}+\frac{m(z)_{22}-(1 / 2 \varepsilon) \operatorname{det}(m(z))}{z+\varepsilon}=0 .
$$

Now, $m(z)=C_{0}\left(B_{0}-z\right)^{-1} C_{0}^{*}$ is positive and increasing for $z \in\left(-\infty, \lambda_{0,1}^{0}-\eta\right)$. As $0<m(z)_{i i}=0\left(\alpha^{2}\right)$, while $0<\operatorname{det}(m(z))=0\left(\alpha^{4}\right)$, both numerators are positive at $z=\mp \varepsilon$. The graph of the function in the 1.h.s. is schematically depicted in Fig.1 (for the case $\left.\varepsilon<\lambda_{0,1}^{0}-\eta\right)$.

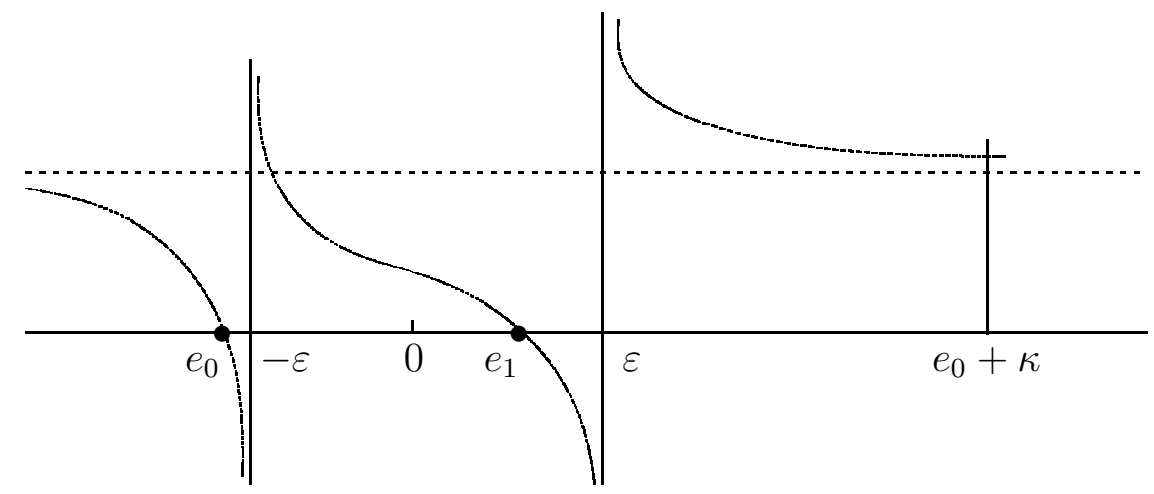

Figure 1a: The graph of the 1.h.s. of (3.3) : case of two roots

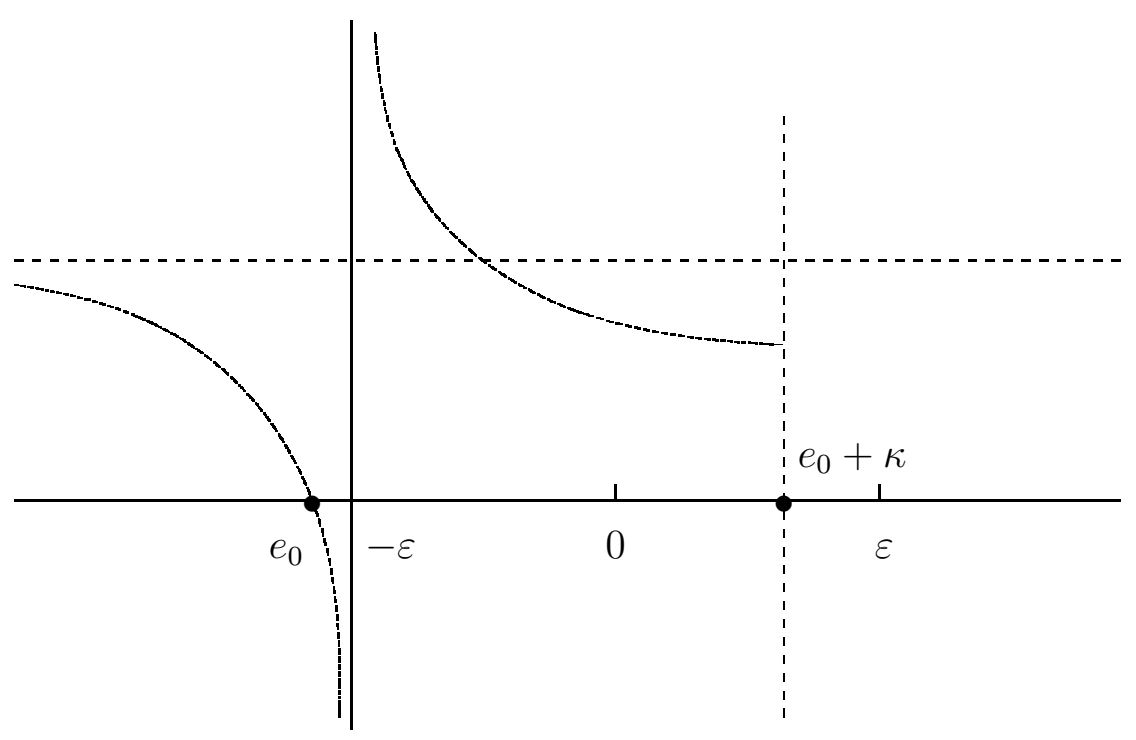

Figure 1b: The graph of the 1.h.s. of (3.3) : case of one root 
One concludes that there exists always a zero $e_{0}<-\varepsilon$. Also, whenever $\varepsilon<\lambda_{0,1}^{0}-\eta=$ $-\varepsilon+\kappa-\eta$, there exists a second zero $-\varepsilon<e_{1}<\varepsilon$. For $z=e_{i}, i=0,1$, Eq.(3.1) with $g=0$ has nontrivial normalized solutions $f_{i}$, which can be completed with the higher components $-M_{0}\left(e_{i}\right) f_{i}$ to eigenvectors of $H$ :

$$
F_{0}^{(i)}(Q)= \begin{cases}f_{i} & , Q=\emptyset \\ \left(-M_{0}\left(e_{i}\right) f_{i}\right)(Q) & , Q \neq \emptyset\end{cases}
$$

Eq.(3.2) has no other real solution $z<\lambda_{0,1}^{0}-\eta$. Also, there are no complex solutions $\tilde{z}, \operatorname{Im} \tilde{z} \neq 0$ : otherwise, considering an eigenvector $\tilde{f}$ of the matrix $\varepsilon \sigma_{3}-m(\tilde{z})$ corresponding to the eigenvalue $\tilde{z}$ and completing it with the vector $-M_{0}(\tilde{z}) \tilde{f} \in \mathcal{H}_{>0}$, one would obtain an eigenvector of $H$ with a non-real eigenvalue.

$>$ From now on, we consider for definiteness the case in which Eq. (3.3) has two solutions $e_{0}, e_{1}<\lambda_{0,1}^{0}-\eta$. The case with one solution is treated similarly.

II. For $n=1$, the equation (2.9) is a system of two equations, valid for $z \in D_{1, \eta}$ :

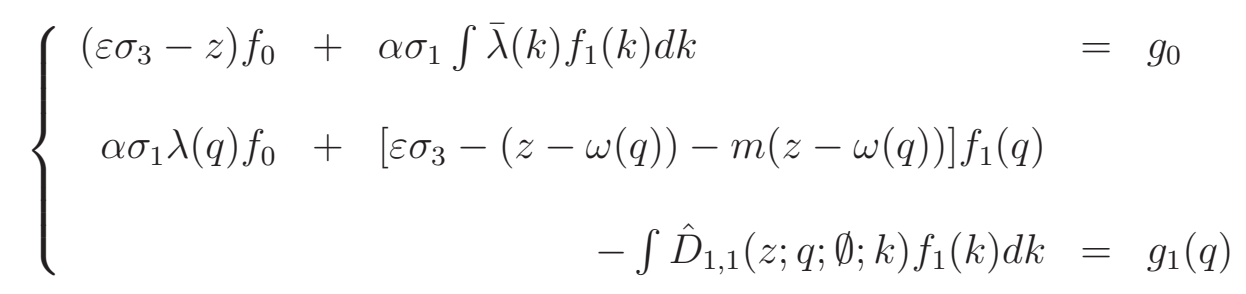

The operator

$$
\left[B(z) f_{1}\right](q)=\left[\varepsilon \sigma_{3}-z+\omega(q)-m(z-\omega(q))\right] f_{1}(q)-\int \hat{D}_{1,1}(z ; q ; \emptyset ; k) f_{1}(k) d k
$$

has an analytic inverse whenever the matrices $\varepsilon \sigma_{3}-(z-\omega(q))-m(z-\omega(q)), \forall q \in \mathbb{R}^{d}$ are invertible, what happens for $z \in \mathbb{C} \backslash I$, where we denoted $I=\left[e_{0}+\kappa, \infty\right)$. As we shall see below, $\lambda_{0,1}=e_{0}+\kappa$ is the left boundary of the spectrum of $H$. Let $\bar{D}_{0}=\{z \in \mathbb{C}$ : $\left.\operatorname{Re} z<\lambda_{0,1}\right\}, n=1,2, \ldots$. The following proposition holds:

Proposition 3.1 For $\eta>0$ sufficiently small, $\alpha<\alpha_{0}(\eta, 1)$ and $z \in D_{1, \eta} \backslash I$ the inverse $B(z)^{-1}$ exists and has the representation

$$
\begin{aligned}
{\left[B(z)^{-1} f\right](q) } & =\left[\varepsilon \sigma_{3}-(z-\omega(q))-m(z-\omega(q))\right]^{-1} \\
& \times\left\{f(q)+\int K(z ; q, k)\left[\varepsilon \sigma_{3}-(z-\omega(k))-m(z-\omega(k))\right]^{-1} f(k) d k\right\}
\end{aligned}
$$

where the kernel $K(z ; \cdot, \cdot) \in \mathcal{K}_{1}$ and its $\mathcal{K}_{1}$-norm is uniformly bounded for $z \in \bar{D}_{0}$. Besides, $K(z ; \cdot, \cdot)$ is a $\mathcal{K}_{1}$-valued analytic function of $z$ in $D_{1, \eta} \backslash I$, which has boundary values at the cut $I$, i.e. for all $x \in\left[e_{0}+\kappa, \lambda_{0,2}^{0}-\eta\right)$ the following limits exist in $\mathcal{K}_{1}$ :

$$
K^{ \pm}(x ; \cdot, \cdot)=\lim _{\epsilon \searrow 0} K(x \pm i \epsilon ; \cdot, \cdot) .
$$

The kernels $K^{ \pm}(x ; \cdot, \cdot)$ are Hölder-continuous $\mathcal{K}_{1}$-valued functions of $x \in\left[e_{0}+\kappa, \lambda_{0,2}^{0}-\eta\right)$. 
A similar statement is outlined in our paper [1]. For the reader convenience below we shortly resume the proof of the Proposition 3.1 and we leave a detailed demonstration (which includes a generalization of the Privalov lemma) for Appendix. Note that essentially the proof consists in the following :

Denoting by $\hat{D}_{1,1}$ the integral operator with the kernel $\hat{D}_{1,1}(z ; q ; \emptyset ; k) \in \mathcal{K}_{1}$, one has:

$$
\begin{aligned}
B(z)^{-1} & =\left(\varepsilon \sigma_{3}-(z-\omega(\cdot))-m(z-\omega(\cdot))\right)^{-1} \\
& +\left\{\sum_{k=1}^{\infty}\left[\left(\varepsilon \sigma_{3}-(z-\omega(\cdot))-m(z-\omega(\cdot))\right)^{-1} \hat{D}_{1,1}\right]^{k}\right\} \\
& \times\left(\varepsilon \sigma_{3}-(z-\omega(\cdot))-m(z-\omega(\cdot))\right)^{-1},
\end{aligned}
$$

where every term of the sum is an integral operator with kernel in $\mathcal{K}_{1}$ and the sum converges in $\mathcal{K}_{1}$ for $\alpha$ sufficiently small, uniformly for $z \in D_{1, \eta}$. Hence we arrive at the representation (3.7), where the kernel $K(z ; \cdot, \cdot) \in \mathcal{K}_{1}$ depends analytically on $z \in$ $D_{1, \eta} \backslash I$. The existence of the limits (3.8) and the properties of the boundary value kernels $K^{ \pm}(x ; \cdot, \cdot)$ are proved for every term of the series (3.9) using induction over $k$. Thereby, we use that, if two $z$-dependent kernels $K_{1}(z), K_{2}(z) \in \mathcal{K}_{1}$ possess boundary values like in Eq.(3.8), then the kernel

$$
K_{3}(z ; q, k):=\int K_{2}\left(z ; q, q^{\prime}\right)\left[\varepsilon \sigma_{3}-\left(z-\omega\left(q^{\prime}\right)\right)-m\left(z-\omega\left(q^{\prime}\right)\right)\right]^{-1} K_{1}\left(z ; q^{\prime}, k\right) d q^{\prime},
$$

has the same property, in view of the Sokhotski formula: $1 /(x+i 0)=\mathcal{P}(1 / x)+i \pi \delta(x)$. Indeed, the inverse matrix $\left(\varepsilon \sigma_{3}-\left(z-\omega\left(q^{\prime}\right)\right)-m\left(z-\omega\left(q^{\prime}\right)\right)\right)^{-1}$ has the structure: either

$$
\frac{A_{0}}{e_{0}-\left(z-\omega\left(q^{\prime}\right)\right)}+\phi\left(z-\omega\left(q^{\prime}\right)\right)
$$

if Eq. (3.2) has one solution $e_{0}$; or

$$
\frac{A_{0}}{e_{0}-\left(z-\omega\left(q^{\prime}\right)\right)}+\frac{A_{1}}{e_{1}-\left(z-\omega\left(q^{\prime}\right)\right)}+\phi\left(z-\omega\left(q^{\prime}\right)\right),
$$

if a second solution $e_{1}$ exists, too. Here, $A_{0}, A_{1}$ are $2 \times 2$-matrices and $\phi(z)$ is a $\mathcal{M}_{2}$-valued analytic function in $D_{0, \eta}$. Therefore, denoting by $d \nu_{x}(q)$ the Gelfand-Leray measure on the surface $\mathcal{C}_{1, y}=\left\{q^{\prime}: \omega\left(q^{\prime}\right)=y\right\}$, we obtain

$$
\begin{aligned}
K_{ \pm}(x ; q, k)= & \int_{\kappa}^{\infty} d y\left[\left(\sum_{j=0,1} A_{j} /\left[e_{j}+y-x \pm i 0\right]+\phi(x-y)\right)\right. \\
& \left.\times \int_{\mathcal{C}_{1, y}} K_{1, \pm}\left(x ; q ; q^{\prime}\right) K_{2, \pm}\left(x ; q^{\prime} ; k\right) d \nu_{y}\left(q^{\prime}\right)\right] d x
\end{aligned}
$$

As the internal integral over the surface $\mathcal{C}_{1, y}$ is a smooth function of $y$, the integral with respect to $y$ can be done and gives Hölder continuous functions of $x, K_{i, \pm}(x ; \cdot, \cdot), i=1,2$ (as follows from the Plemelj-Privalov theorem [15], [16], and Appendix). 
Next, we consider Eq.(3.5) for $g_{1}=0$, i.e. again the resolvent of $H$ restricted to $\mathcal{H}_{0}$. We have

$$
f_{1}(\cdot)=-\alpha(B(z))^{-1} \sigma_{1} \lambda(\cdot) f_{0}
$$

Plugging $f_{1}$ into the first equation (3.5) we obtain that

$$
\left(\varepsilon \sigma_{3}-z-\alpha^{2} \sigma_{1} \int \bar{\lambda}(q)\left[B(z)^{-1} \sigma_{1} \lambda(\cdot)\right](q) d q\right) f_{0}=g_{0}
$$

Comparing this with Eq. (3.1) we obtain:

$$
m_{0}(z ; \emptyset)=\alpha^{2} \sigma_{1} \int \bar{\lambda}(q)\left[B(z)^{-1} \sigma_{1} \lambda(\cdot)\right](q) d q
$$

what provides the analytic continuation of $m(z)$ to $D_{1, \eta} \backslash I$. We have thus shown that Eq. (3.3) determines completely the discrete spectrum of the operator $H$ below its continuous spectrum.

Let us remark that $\lim _{\xi \int_{0}+\kappa} m(\xi)$ is finite in dimension $d \geq 3$, because the $1 / q^{2}$ singularity of the integrand in Eq.(3.7) is integrable. Therefore, in the case $\varepsilon>e_{0}+\kappa$, the second solution $e_{1}$ of Eq.(3.3) exists if, and only if, the 1.h.s. of that equation is negative for $\xi=e_{0}+\kappa$. This exhausts the discrete spectrum of $H$ below its continuous spectrum.

\section{One-boson branches}

We proceed now to the construction of the one-particle branches of the (continuous) spectrum of the operator $H$. Consider the family $\left\{A(\xi), \xi \in\left[e_{0}+\kappa, \lambda_{0,2}^{0}-\eta\right]\right\}$ of selfadjoint operators, acting in $\mathcal{H}_{\leq 1}$ according to: for $F=\left(f_{0}, f_{1}\right) \in \mathcal{H}_{\leq 1}$,

$$
\begin{aligned}
& (A(\xi) F)_{0} \quad=\quad \varepsilon \sigma_{3} f_{0} \quad+\alpha \sigma_{1} \int \bar{\lambda}(k) f_{1}(k) d k \\
& (A(\xi) F)_{1}(q)=\alpha \sigma_{1} \lambda(q) f_{0}+\left(\varepsilon \sigma_{3}+\omega(q)-m(\xi-\omega(q))\right) f_{1}(q) \\
& -\int \hat{D}_{1,1}(\xi ; q, \emptyset, k) f_{1}(k) d k
\end{aligned}
$$

Along with this, consider the family $\left\{A_{0}(\xi), \xi \in\left[e_{0}+\kappa, \lambda_{2}^{0}-\eta\right]\right\}$ acting in $\mathcal{H}_{1}$ :

$$
\left(A_{0}(\xi) f\right)(q)=\left(\varepsilon \sigma_{3}+\omega(q)-m(\xi-\omega(q))\right) f(q), \quad f \in \mathcal{H}_{1} .
$$

Concerning the latter, let us denote by $e_{0}(\xi, q), e_{1}(\xi, q)$ the eigenvalues of the matrix $\varepsilon \sigma_{3}+\omega(q)-m(\xi-\omega(q))$. One can easily see that these eigenvalues are the solutions of the equation

$$
1+\frac{m_{11}(\xi-\omega(q))+\frac{1}{2 \varepsilon} \operatorname{det} m(\xi-\omega(q))}{-\varepsilon+\omega(q)-e}+\frac{m_{22}(\xi-\omega(q))-\frac{1}{2 \varepsilon} \operatorname{det} m(\xi-\omega(q))}{\varepsilon+\omega(q)-e}=0
$$


which is similar to Eq. (3.3) . The graph of the 1.h.s. as a function of $e$ looks like the graph of Fig. 1.

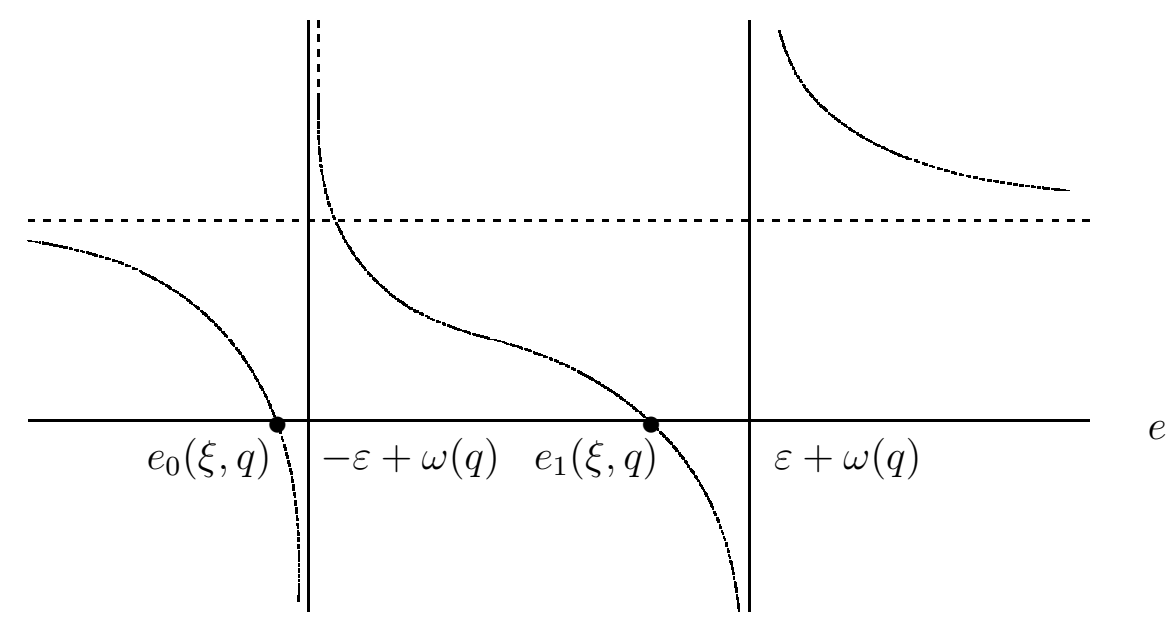

Figure 2: The graph of the 1.h.s. of (4.3)

Hence one can see that its roots are simple and placed in the order $e_{0}(\xi, q)<\omega(q)-\varepsilon<$ $e_{1}(\xi, q)<\omega(q)+\varepsilon$. As, for fixed $q$, the matrix $m(\xi-\omega(q))$ is positive and increasing of $\xi<\lambda_{2}^{0}-\eta$, both roots $e_{0}(\xi, q), e_{1}(\xi, q)$ are monotonously decreasing functions of $\xi$ in the interval $\left(-\infty, \lambda_{0,2}^{0}-\eta\right)$. Their graphs are sketched in Fig. 2. Moreover, as a consequence of Eq. (4.3), the two roots belong respectively to $O\left(\alpha^{2}\right)$-neighbourhoods of $\omega(q) \mp \varepsilon$, therefore the distance between them is larger than $\varepsilon$ for small $\alpha$ :

$$
e_{1}(\xi, q)-e_{0}(\xi, q)>\varepsilon
$$

For every $\xi \in\left[e_{0}+\kappa, \lambda_{0,2}^{0}-\eta\right)$, let

$$
E(\xi)=\inf _{q} e_{0}(\xi, q)=-\varepsilon+\kappa-O\left(\alpha^{2}\right) .
$$

Obviously, the spectrum of $A_{0}(\xi)$ is absolutely continuous and covers the half-axis $[E(\xi), \infty)$.

Let us now calculate the resolvent $(A(\xi)-z)^{-1}$ of $A(\xi)$. As a preliminary to this, we consider the resolvent of the operator $\tilde{B}(\xi)$ acting in $\mathcal{H}_{1}$ as

$$
(\tilde{B}(\xi))(q)=\left[\varepsilon \sigma_{3}+\omega(q)-m(\xi-\omega(q))\right] f_{1}(q)-\int \hat{D}_{1,1}(\xi ; q ; \emptyset ; k) f_{1}(k) d k ; f \in \mathcal{H}_{1} .
$$

In the same way as for Eq. (3.7), we find for $R_{\tilde{B}(\xi)}(z)=(\tilde{B}(\xi)-z)^{-1}$

$$
\begin{aligned}
\left(R_{\tilde{B}(\xi)}(z) g\right)(q) & \left.=\left[\varepsilon \sigma_{3}-z+\omega(q)\right)-m(\xi-\omega(q))\right]^{-1} \\
& \times\left\{g(q)+\int K_{\xi}(z ; q, k)\left[\varepsilon \sigma_{3}-z+\omega(k)-m(\xi-\omega(k))\right]^{-1} g(k) d k\right\}
\end{aligned}
$$


where the kernel $K_{\xi}(z ; \cdot, \cdot) \in \mathcal{K}_{1}$ is a $\mathcal{K}_{1}$-analytic function of $z$ in $\mathbb{C} \backslash[E(\xi), \infty)$. Thereby, the limits

$$
K_{\xi}^{ \pm}(x ; \cdot, \cdot)=\lim _{\epsilon \searrow 0} K_{\xi}(x+i \epsilon ; \cdot, \cdot)
$$

exist in $\mathcal{K}_{1}$ and the functions $K_{\xi}^{ \pm}(x ; \cdot, \cdot)$ are $\mathcal{K}_{1}$-valued Hölder continuous functions of $x \in[E(\xi), \infty)$.

The resolvent $R_{A(\xi)}(z)=\left(A(\xi)-z \mathbb{I}_{\leq 1}\right)^{-1}$ can now be written as: for $G=\left(g_{0}, g_{1}\right)$,

$$
\begin{aligned}
\left(R_{A(\xi)}(z) G\right)_{0} & =f_{0}=\Delta_{\xi}^{-1}(z)\left(g_{0}-\alpha \sigma_{1} \int\left(R_{\tilde{B}(\xi)}(z) g_{1}\right)(k) \bar{\lambda}(k) d k\right) \\
\left(R_{A(\xi)}(z) G\right)_{1}(q) & =f_{1}(q)=\left(R_{\tilde{B}(\xi)}(z)\left[g_{1}(\cdot)-\alpha \sigma_{1} \lambda(\cdot) f_{0}\right]\right)(q)
\end{aligned}
$$

where $\Delta_{\xi}(z)$ denotes the $\mathcal{M}_{2}$-valued analytic function of $z \in \mathbb{C} \backslash[E(\xi), \infty)$

$$
\Delta_{\xi}(z)=\varepsilon \sigma_{3}-z-\alpha^{2} \sigma_{1} \int\left[R_{\tilde{B}(\xi)}(z) \lambda(\cdot)\right](k) \bar{\lambda}(k) d k
$$

The inverse matrix $\Delta_{\xi}^{-1}(z)$ has either one, or two simple poles $\tau_{0}(\xi)<\tau_{1}(\xi)<E(\xi)$ lying on the real axis at the left of $-\varepsilon$ and $\varepsilon$, respectively. These poles are the eigenvalues of the operator $A(\xi)$ with corresponding eigenvectors $\psi_{\xi}^{(0)}, \psi_{\xi}^{(1)}$ :

$$
\psi_{\xi}^{(i)}=\left(f_{\xi, 0}^{(i)}, f_{\xi, 1}^{(i)}(q)=-\alpha\left(R_{\tilde{B}(\xi)}\left(\tau_{i}(\xi)\right) \sigma_{1} \lambda(\cdot)\right)(q) f_{\xi, 0}^{(i)}\right), i=0,1
$$

where $f_{\xi, 0}^{(i)}$ are the null vectors of the matrix $\Delta_{\xi}\left(\tau_{i}(\xi)\right)$. As agreed before, we consider the case of two roots $\tau_{0}(\xi), \tau_{1}(\xi)$. Also, the limits

$$
\left[\Delta_{\xi}^{-1}(x)\right]^{ \pm}=\lim _{\epsilon \searrow 0} \Delta_{\xi}^{-1}(x \pm i \epsilon), x \in[E(\xi), \infty)
$$

exist, whereby, for $\alpha$ small,

$$
\pm \operatorname{Im}\left[\Delta_{\xi}^{ \pm}(x)\right]=\alpha^{2} \pi \sigma_{1}\left(\begin{array}{cc}
\int_{\omega(k)-\varepsilon=x}|\lambda(k)|^{2} d k & 0 \\
0 & \int_{\omega(k)+\varepsilon=x}|\lambda(k)|^{2} d k
\end{array}\right)+O\left(\alpha^{4}\right) .
$$

Hence, as a consequence of assumption (A3), the matrices $\Delta_{\xi}^{ \pm}(x)^{-1}$ are non-singular on $[E(\xi), \infty)$, in other words, the operator $A(\xi)$ has no eigenvalues embedded in the continuous spectrum.

Remark that, in view of Eq. (3.10), for $z=\xi$, the matrix $\Delta_{\xi}(z)$ equals the matrix $\varepsilon \sigma_{3}-\xi-m(\xi)$ in the l.h.s. of Eq. (3.1) and the zeros $\tau_{i}(\xi)$ of $\operatorname{det} \Delta_{\xi}(z)$ satisfy

$$
\tau_{i}(\xi)=\xi, i=0,1
$$

i.e. the condition under which they equal, respectively, the solutions $e_{i}, i=0,1$ of Eq. (3.3). 
Let us construct, for every $\xi \in\left(e_{0}+\kappa, \lambda_{0,2}^{0}-\eta\right)$, the vectors

$$
F_{\xi}^{(i)}=\left(\psi_{\xi}^{(i)}, \bar{\psi}_{\xi}^{(i)}=M_{1}(\xi) f_{\xi, 1}^{(i)}\right) \in \mathcal{H}
$$

which, obviously, fulfill the equation

$$
H F_{\xi}^{(i)}=\xi F_{\xi}^{(i)}+\left(\tau_{i}(\xi)-\xi\right) \psi_{\xi}^{(i)},
$$

therefore, if $\xi=e_{i}$ they are the eigenvectors of $H$ with eigenvalues $e_{i}, i=0,1$. In this way, we reobtain the already constructed eigenvectors of $H$, Eq. (3.4).

Using general criteria of absence of the singular continuous spectrum of a self-adjoint operator [18] and the explicit form of the resolvent $R_{A(\xi)}(z)$, one can show that $A(\xi)$ has no singular spectrum. Hence, the space $\mathcal{H}_{\leq 1}$ splits into the orthogonal sum of two invariant subspaces of $A(\xi)$ :

$$
\mathcal{H}_{\leq 1}=\mathcal{H}^{\mathrm{ac}}+\mathcal{H}^{\mathrm{d}}
$$

where $\mathcal{H}^{\mathrm{ac}}=\mathcal{H}^{\mathrm{ac}}(\xi)$ is the subspace of absolute continuity of $A(\xi)$ and $\mathcal{H}^{\mathrm{d}}=\mathcal{H}^{\mathrm{d}}(\xi)$ is the subspace corresponding to the discrete spectrum, generated by the vectors $\psi_{\xi}^{(0)}, \psi_{\xi}^{(1)}$ (or by $\psi_{\xi}^{(0)}$ alone in the case of one root).

Consider the embedding

$$
\mathcal{I}: \mathcal{H}_{1} \rightarrow \mathcal{H}_{\leq 1}: \quad \mathcal{I} f=(0, f) \in \mathcal{H}_{\leq 1} \text { for } f \in \mathcal{H}_{1}
$$

and construct the wave operator

$$
s-\lim _{t \rightarrow+\infty} \exp [i t A(\xi)] \mathcal{I} \exp \left[-i t A_{0}(\xi)\right]=\Omega^{+}=\Omega^{+}(\xi)
$$

The limit exists and $\Omega^{+}: \mathcal{H}_{1} \rightarrow \mathcal{H}_{\leq 1}$ is unitary and

$$
\Omega^{+} A_{0}(\xi)\left(\Omega^{+}\right)^{-1}=\left.A(\xi)\right|_{\mathcal{H}^{\mathrm{ac}}}
$$

i.e. the restriction of $A(\xi)$ to $\mathcal{H}^{\text {ac }}$ is unitarily equivalent to $A_{0}(\xi)$ (for details, see [1] or [13]).

The generalized eigenfunctions of the operator $A_{0}(\xi)$ have the form

$$
\delta_{\bar{q}, \xi}^{(i)}=\delta(q-\bar{q}) \phi_{i}(\xi, \bar{q})
$$

where $\phi_{i}(\xi, \bar{q})$ are the eigenvectors of the matrix $\varepsilon \sigma_{3}+\omega(\bar{q})-m(\xi-\omega(\bar{q}))$ corresponding to the eigenvalues $e_{i}(\xi, \bar{q}), i=0,1$ - the solutions of the equation

$$
\operatorname{det}\left[\varepsilon \sigma_{3}-z+\omega(\bar{q})-m(\xi-\omega(\bar{q}))\right]=0 .
$$

Known formulas of scattering theory ([17]) allow to write the generalized eigenfunctions of the continuous spectrum of $A(\xi)$ as

$$
\psi_{\bar{q}, \xi}^{(i)}=\Omega^{+} \delta_{\bar{q}, \xi}^{(i)}=\lim _{\epsilon \searrow 0} i \epsilon R_{A(\xi)}\left(e_{i}(\xi, \bar{q})-i \epsilon\right) \mathcal{I} \delta_{\bar{q}, \xi}^{(i)}
$$


Using the explicit form (4.7) of the resolvent $R_{A(\xi)}(z)$, we find

$$
\begin{array}{r}
\psi_{\bar{q}, \xi, 0}^{(i)}=\begin{array}{r}
-\alpha \Delta_{\xi}^{-1}\left(e_{i}(\xi, \bar{q})\right) \sigma_{1}\left[\bar{\lambda}(\bar{q})+\int\left(\varepsilon \sigma_{3}-e_{i}(\xi, \bar{q})+\omega(k)-m(\xi-\omega(k))+i 0\right)^{-1}\right. \\
\left.\times K^{-}\left(e_{i}(\xi, \bar{q}) ; k, \bar{q}\right) \bar{\lambda}(k) d k\right] \phi_{i}(\xi, \bar{q})
\end{array} \\
\psi_{\bar{q}, \xi, 1}^{(i)}(q)= \\
\delta(q-\bar{q}) \phi_{i}(\xi, \bar{q})+\left(\varepsilon \sigma_{3}-e_{i}(\xi, \bar{q})+\omega(q)-m(\xi-\omega(q)+i 0)\right)^{-1} \\
\times K^{-}\left(e_{i}(\xi, \bar{q}) ; q, \bar{q}\right) \phi_{i}(\xi, \bar{q})
\end{array}
$$

The somewhat formal derivation of the form of the generalized functions $\psi_{\bar{q}, \xi}^{(i)}$ is bolstered by the following lemma:

\section{Lemma 4.1}

(a) For every fixed $\bar{q} \in \mathbb{R}^{d}, \xi<\lambda_{0,2}^{0}-\eta$ and $i=0,1$ :

i. the vector $\psi_{\bar{q}, \xi, 0}^{(i)} \in \mathbb{C}^{2}$ is a $\mathbb{C}^{2}$-valued bounded function of $\bar{q}$;

(ii.) the function $\psi_{\bar{q}, \xi, 1}^{(i)}(q)$ is a $\mathbb{C}^{2}$-valued generalized function of $q$ in $\tilde{\tilde{\mathcal{B}}}_{1}^{\prime}$ and, for every fixed $q \in \mathbb{R}^{d}$, it is a $\mathbb{C}^{2}$-valued generalized function of $\bar{q}$ in $\tilde{\tilde{\mathcal{B}}}_{1}^{\prime}$.

(b) For any $\varphi \in \mathcal{S}\left(\mathbb{R}^{d}\right)$, define

$$
C_{\varphi, \xi, 0}^{(i)}=\int \varphi(\bar{q}) \psi_{\bar{q}, \xi, 0}^{(i)} d \bar{q}, \quad C_{\varphi, \xi, 1}^{(i)}(q)=\int \varphi(\bar{q}) \psi_{\bar{q}, \xi, 1}^{(i)}(q) d \bar{q}
$$

then,

$$
\Psi_{\varphi, \xi}^{(i)}=\left(C_{\varphi, \xi, 0}^{(i)}, C_{\varphi, \xi, 1}^{(i)}(\cdot)\right) \in \mathcal{H}_{\leq 1} .
$$

Thereby, for two functions $\varphi_{1}, \varphi_{2} \in \mathcal{S}\left(\mathbb{R}^{d}\right)$,

$$
\begin{aligned}
\left(\Psi_{\varphi_{1}, \xi}^{(i)}, \Psi_{\varphi_{2}, \xi}^{\left(i^{\prime}\right)}\right) & =\left(C_{\varphi_{1}, \xi, 0}^{(i)}, C_{\varphi_{2}, \xi, 0}^{\left(i^{\prime}\right)}\right)_{\mathbb{C}^{2}}+\int\left(C_{\varphi_{1}, \xi, 1}^{(i)}(q), C_{\varphi_{2}, \xi, 1}^{\left(i^{\prime}\right)}(q)\right)_{\mathbb{C}^{2}} d q \\
& =\left(\varphi_{1}, \varphi_{2}\right)_{L_{2}\left(\mathbb{R}^{d}\right)} \delta_{i, i^{\prime}}
\end{aligned}
$$

A similar lemma appears in [1] and the proof there applies in our case. The meaning of relation (4.17) can be better seen by writing it more formally:

$$
\left(\Psi_{\bar{q}, \xi}^{(i)}, \Psi_{\bar{q}^{\prime}, \xi}^{\left(i^{\prime}\right)}\right)=\delta\left(\bar{q}-\bar{q}^{\prime}\right) \delta_{i, i^{\prime}}
$$

In this way, for every $\xi<\lambda_{0,2}^{0}-\eta$ and $\bar{q} \in \mathbb{R}^{d}$, we constructed two generalized eigenvectors $\Psi_{\bar{q}, \xi}^{(i)} \in \tilde{\tilde{\mathcal{B}}}_{\leq 1}^{\prime}, i=0,1$ of the operator $A(\xi)$ with the eigenvalues $e_{i}(\bar{q}, \xi), i=0,1$, respectively. By acting on them with the operator $M_{1}(\xi)$ (see Remark 2.5) we obtain the generalized functions

$$
\bar{\Psi}_{\bar{q}, \xi}^{(i)}=M_{1}(\xi) \Psi_{\bar{q}, \xi}^{(i)} \in \tilde{\tilde{\mathcal{B}}}_{>1}^{\prime} .
$$


Obviously, the entire vector $F_{\bar{q}, \xi}^{(i)}=\left\{\Psi_{\bar{q}, \xi}^{(i)}, \bar{\Psi}_{\bar{q}, \xi}^{(i)} \in \tilde{\tilde{\mathcal{B}}}^{\prime}\right\}$ satisfies the equation

$$
H F_{\bar{q}, \xi}^{(i)}=\xi F_{\bar{q}, \xi}^{(i)}+\left(e_{i}(\bar{q}, \xi)-\xi\right) \Psi_{\bar{q}, \xi}^{(i)}
$$

(here $H$ denotes the extension of $H$ to $\tilde{\mathcal{B}^{\prime}}$, cf. Remark 2.5) . Let $\xi^{(i)}(\bar{q}), i=0,1$ denote the unique (as seen on Fig. 3) solutions less than $\pm \varepsilon$ respectively, of the equations

$$
e_{i}(\bar{q}, \xi)=\xi, \quad i=0,1
$$

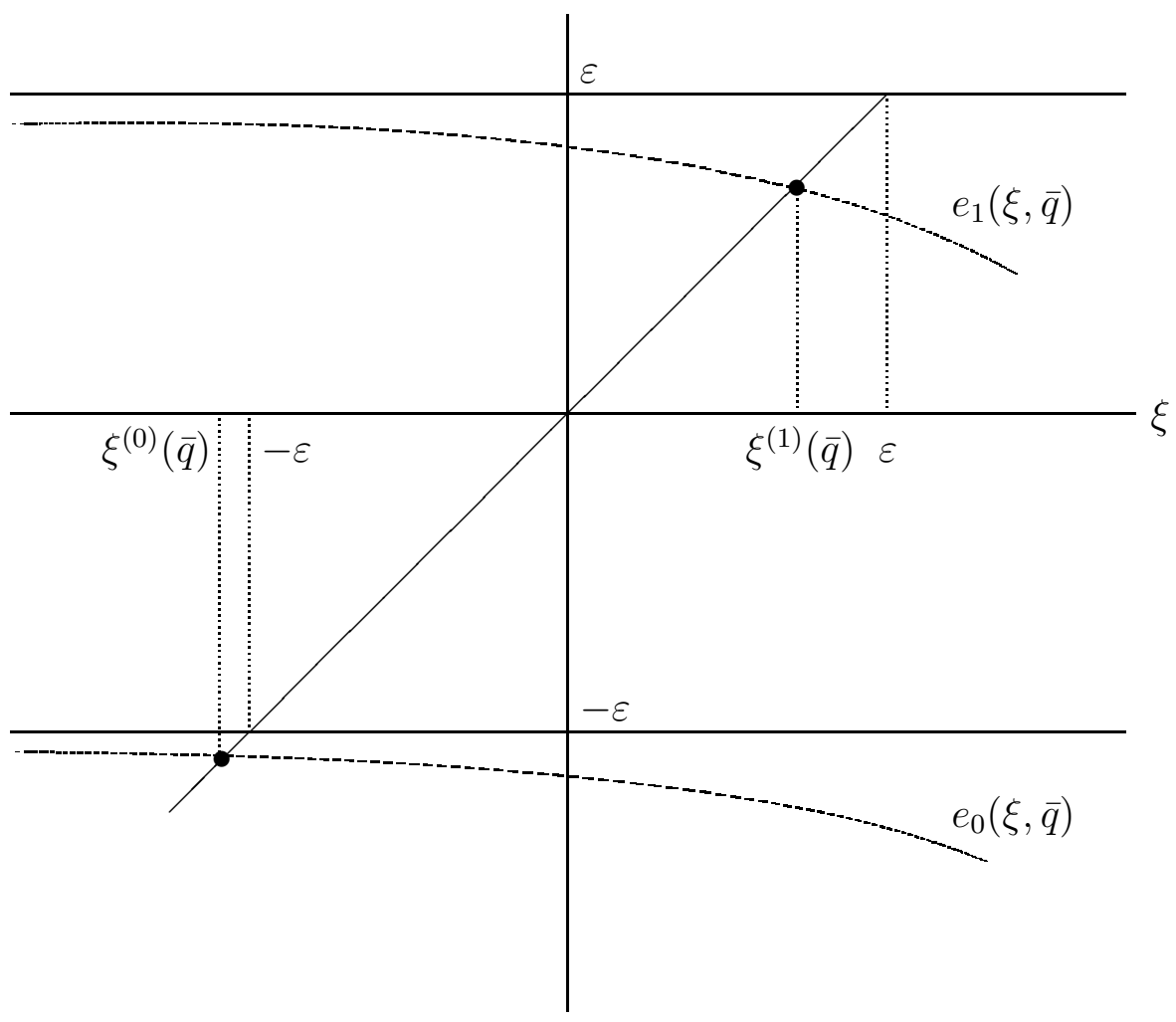

Figure 3: Solving Eq. 4.20)

Now, it is clear that $F_{\bar{q}, \xi=\xi^{(i)}(\bar{q})}^{(i)}=F_{\bar{q}}^{(i)}$ is a generalized eigenvector of $H$ with eigenvalue $\xi^{(i)}(\bar{q})$. We remind that $e_{i}(\bar{q}, \xi)$ satisfies Eq.(4.14), which, for $z=\xi$, becomes

$$
\operatorname{det}\left[\varepsilon \sigma_{3}-(\xi-\omega(q))-m(\xi-\omega(q))\right]=0 \text {. }
$$

Comparing this equation with Eq. (3.2), we see that Eq. (4.21) has two solutions:

$$
\xi^{(i)}(\bar{q})=e_{i}+\omega(q)
$$


where $e_{i}, i=0,1$ are the two solutions of Eq.(3.2). Thereby, as the matrix $\varepsilon \sigma_{3}+\omega(\bar{q})-$ $m(\xi-\omega(\bar{q}))$ approaches, when $\xi \rightarrow \xi^{(i)}(\bar{q})$ the matrix $\varepsilon \sigma_{3}+\omega(q)-m\left(e_{i}\right)$, one of its eigenvectors $\phi_{i}(\xi, \bar{q})$ approaches the eigenvector of the latter for the eigenvalue $\xi^{(i)}(\bar{q})$, i.e. for all $\bar{q}$ and $i=0,1,\left.\quad \varphi_{i}(\xi, \bar{q})\right|_{\xi=\xi^{(i)}(\bar{q})}=f_{i}$.

The conditions

$$
\xi^{(i)}(\bar{q})<\lambda_{0,2}^{0}-\eta, \quad \xi^{(0)}(\bar{q})<\xi^{(1)}(\bar{q})
$$

define two bounded domains $G_{\eta}^{(i)} \subset \mathbb{R}^{d}$ of allowed values of $\bar{q}$ :

$$
\begin{array}{rc}
G_{\eta}^{(0)} & =\quad\left\{\bar{q}: \xi^{(0)}(\bar{q})<\lambda_{0,2}^{0}-\eta\right\} \\
G_{\eta}^{(1)} & =\left\{\bar{q}: \xi^{(1)}(\bar{q})<\lambda_{0,2}^{0}-\eta\right\} \subset G_{\eta}^{(0)} .
\end{array}
$$

For $\bar{q} \in G_{\eta}^{(1)}$, there are two generalized eigenvectors of $H, F_{\bar{q}}^{(i)}=F_{\bar{q}, \xi^{(i)}(\bar{q})}^{(i)}, i=0,1$, with eigenvalues $\xi^{(i)}(\bar{q}), i=0,1$, respectively, while, for $\bar{q} \in G_{\eta}^{(0)} \backslash G_{\eta}^{(1)}$, there is only one eigenvector left. The functions $\xi^{(i)}(\bar{q})$ are smooth functions of $\bar{q} \in G_{\eta}^{(i)}$ and $\min _{\bar{q}} \xi^{(0)}(\bar{q})=$ $e_{0}+\kappa$ coincides with the lowest end of the continuous spectrum of $H$.

Let $\mathcal{C}_{\infty}^{0}\left(G_{\eta}^{(i)}\right)=: \mathcal{C}^{(i)}, i=0,1$ be the space of infinitely smooth functions with support in the domains $G_{\eta}^{(i)}, i=0,1$, respectively.

Lemma 4.2 For any $\varphi \in \mathcal{C}^{(i)}$, the vector

$$
F_{\varphi}^{(i)}=\int_{G_{\eta}^{(i)}} F_{\bar{q}}^{(i)} \varphi(\bar{q}) d \bar{q} \in \mathcal{H} .
$$

A similar statement is contained in [1] and the proof there applies to the case at hand through verbatim.

We introduce now two subspaces of $\mathcal{H}$ as the closures of the linear span of the vectors (4.24):

$$
\mathcal{H}^{(i)}=\overline{\left\{F_{\varphi}^{(i)}, \varphi \in \mathcal{C}^{(i)}\right\}}, \quad i=0,1 .
$$

As explained in [1], the calculation of the scalar product of two vectors (4.24) reduces to the calculation of the generalized function

$$
Q^{i, i^{\prime}}\left(\bar{q}, \bar{q}^{\prime}\right)=\left(F_{\bar{q}}^{(i)}, F_{\bar{q}^{\prime}}^{\left(i^{\prime}\right)}\right)_{\mathcal{H}}, \bar{q} \in G_{\eta}^{(i)}, \bar{q}^{\prime} \in G_{\eta}^{\left(i^{\prime}\right)} .
$$

As $F_{\bar{q}}^{(i)}, F_{\bar{q}^{\prime}}^{\left(i^{\prime}\right)}$ are generalized eigenvectors of $H$ with eigenvalues $\xi^{(i)}(\bar{q}), \xi^{\left(i^{\prime}\right)}\left(\bar{q}^{\prime}\right)$ respectively, $Q^{i, i^{\prime}}\left(\bar{q}, \bar{q}^{\prime}\right)$ is concentrated on the surface $\left\{\xi^{(i)}(\bar{q})=\xi^{\left(i^{\prime}\right)}\left(\bar{q}^{\prime}\right)\right\}$, hence it is a sum of generalized functions of the form $A(\bar{q}) \delta\left(\bar{q}-\bar{q}^{\prime}\right)$ and $\left.B\left(\bar{q}, \bar{q}^{\prime}\right)\right) \delta\left(\xi^{(i)}(\bar{q})-\xi^{\left(i^{\prime}\right)}\left(\bar{q}^{\prime}\right)\right.$ ) (this is rigorously proved in [1]). Therefore, in calculating $Q^{i, i^{\prime}}\left(\bar{q}, \bar{q}^{\prime}\right)$, one can discard all terms not containing such singularities. So,

$$
\begin{aligned}
Q^{i, i^{\prime}}\left(\bar{q}, \bar{q}^{\prime}\right) & =\left(\psi_{\bar{q}, 0}^{(i)}, \psi_{\bar{q}^{\prime}, 0}^{\left(i^{\prime}\right)}\right)_{\mathbb{C}^{2}}+\int\left(\psi_{\bar{q}, 1}^{(i)}(q), \psi_{\bar{q}^{\prime}, 1}^{\left(i^{\prime}\right)}(q)\right)_{\mathbb{C}^{2}} d q \\
& \left.\left.+\int_{\mathcal{C}>1}\left(\left[M_{1}\left(\xi^{(i)}(\bar{q})\right) \psi_{\bar{q}, 1}^{(i)}(\cdot)\right]\right)(Q),\left[M_{1}\left(\xi^{\left(i^{\prime}\right)}\left(\bar{q}^{\prime}\right)\right) \psi_{\bar{q}^{\prime}, 1}^{\left(i^{\prime}\right)}(\cdot)\right]\right)(Q)\right)_{\mathbb{C}^{2}} d Q
\end{aligned}
$$


(we remind that $\mathcal{C}_{>1}$ denotes the set of all finite subsets with more than one point endowed with the measure (2.2) $)$. It will be convenient to calculate separately the scalar products for each pair $\left(i, i^{\prime}\right)=(0,0),(0,1),(1,1)$ :

- $i=i^{\prime}=0$. The first term in (4.26) is a continuous function of $\bar{q}, \bar{q}^{\prime}$ and will be discarded, as agreed. To calculate the second term in (4.26), we represent $\psi_{\bar{q}, 1}^{(0)}$ as (see (4.15))

$$
\begin{aligned}
\psi_{\bar{q}, 1}^{(0)}(q)= & \delta(q-\bar{q}) f_{0}+\left[A_{0}\left(\xi^{(0)}(\bar{q}), q, \bar{q}\right) f_{0}\right] /\left[\xi^{(0)}(q)-\xi^{(0)}(\bar{q})+i 0\right] \\
+ & {\left[A_{1}\left(\xi^{(0)}(\bar{q}), q, \bar{q}\right) \varphi_{1}^{0}\left(\xi^{(0)}(\bar{q}), q\right)\right] /\left[e_{1}\left(\xi^{(0)}(\bar{q}), q\right)-\xi^{(0)}(\bar{q})+i 0\right] }
\end{aligned}
$$

and similarly $\psi_{\bar{q}^{\prime}, 1}^{(0)}$; here, $f_{0}$ is the null vector of the matrix (3.1) corresponding to the solution $e_{0}$ of Eq. (3.3),$e_{1}\left(\xi^{(0)}(\bar{q}), q\right)$ is the second eigenvalue of the matrix $\varepsilon \sigma_{3}+\omega(q)-m\left(\xi^{(0)}(\bar{q})-\omega(q)\right)$ and $\varphi_{1}^{0}\left(\xi^{(0)}(\bar{q}), q\right)$ the eigenvector corresponding to it, and

$$
\begin{aligned}
& A_{0}\left(\xi^{(0)}(\bar{q}), q, \bar{q}\right)=\left(K^{-}\left(\xi^{(0)}(\bar{q}), q, \bar{q}\right) f_{0}, f_{0}\right)_{\mathbb{C}^{2}} \\
& A_{1}\left(\xi^{(0)}(\bar{q}), q, \bar{q}\right)=\left(K^{-}\left(\xi^{(0)}(\bar{q}), q, \bar{q}\right) \varphi_{1}\left(\xi^{(0)}(\bar{q}), q\right), \varphi_{1}\left(\xi^{(0)}(\bar{q}), q\right)_{\mathbb{C}^{2}}\right.
\end{aligned}
$$

By virtue of Eq. (4.4), the third term in (4.27) is a regular function of $q$ and $\bar{q}$ and we discard it. For the second term we use Sokhotski's formula $1 /[x+i 0]=$ $i \pi \delta(x)+P(1 / x)$ and obtain

$$
\psi_{\bar{q}, 1}^{(0)}(q)=\delta(q-\bar{q}) f_{0}+i \pi A_{0}\left(\xi^{(0)}(\bar{q}), q, \bar{q}\right) \delta\left(\xi^{(0)}(q)-\xi^{(0)}(\bar{q})\right) f_{0}+\text { regular terms }
$$

In this way, the second term in Eq. (4.26) equals, modulo regular terms:

$$
\begin{aligned}
& \int\left(\psi_{\bar{q}, 1}^{(0)}(q), \psi_{\bar{q}^{\prime}, 1}^{(0)}(q)\right)_{\mathbb{C}^{2}} d q=\delta\left(\bar{q}-\bar{q}^{\prime}\right) \\
& +\delta\left(\xi^{(0)}\left(\bar{q}^{\prime}\right)-\xi^{(0)}(\bar{q})\right)\left\{i \pi\left[A_{0}\left(\xi^{(0)}(\bar{q}), \bar{q}^{\prime}, \bar{q}\right)-\overline{A_{0}\left(\xi^{(0)}\left(\bar{q}^{\prime}\right), \bar{q}, \bar{q}^{\prime}\right)}\right]\right. \\
& \left.+\pi^{2} \int_{\xi^{(0)}(q)=\xi^{(0)}(\bar{q})} A_{0}\left(\xi^{(0)}(\bar{q}), q, \bar{q}\right) \overline{A_{0}\left(\xi^{(0)}\left(\bar{q}^{\prime}\right), q, \bar{q}^{\prime}\right)} d q\right\}
\end{aligned}
$$

For the calculation of the third term of Eq. (4.26) we represent $\left(M_{1}\left(\xi^{(0)}(\bar{q})\right) \psi_{\bar{q}, 1}^{(0)}(\cdot)\right)(Q)$ as (see Eq. (2.15)):

$$
\begin{aligned}
\left(M_{1}\left(\xi^{(0)}(\bar{q})\right) \psi_{\bar{q}, 1}^{(0)}(\cdot)\right)(Q) & =\sum_{q \in Q} \mu_{1}^{(0)}\left(\xi^{(0)}(\bar{q}) ; Q \backslash q ; q ; \emptyset\right) \psi_{\bar{q}, 1}^{(0)}(q) \\
& +\int \mu_{1}^{(1)}\left(\xi^{(0)}(\bar{q}) ; Q ; \emptyset ; k\right) \psi_{\bar{q}, 1}^{(0)}(k) d k
\end{aligned}
$$


Now, it is easy to see that the only contribution of this expression to the third term of (4.26) comes from the sum over $q$ and equals

$$
\begin{aligned}
& \int_{\mathbb{R}^{d}} d q \int_{\mathcal{C}_{>1}} d Q\left(\mu_{0}^{(0)}\left(\xi^{(0)}(\bar{q})-\omega(q) ; Q ; \emptyset ; \emptyset\right) f_{0}, \mu_{0}^{(0)}\left(\xi^{(0)}\left(\bar{q}^{\prime}\right)-\omega(q) ; Q ; \emptyset ; \emptyset\right) f_{0}\right)_{\mathbb{C}^{2}} \\
& \times\left[\delta(q-\bar{q})+i \pi A_{0}\left(\xi^{(0)}(\bar{q}) ; q, \bar{q}\right) \delta\left(\xi^{(0)}(q)-\xi^{(0)}(\bar{q})\right)\right] \\
& \times\left[\delta\left(q-\bar{q}^{\prime}\right)+i \pi A_{0}\left(\xi^{(0)}\left(\bar{q}^{\prime}\right) ; q, \bar{q}^{\prime}\right) \delta\left(\xi^{(0)}(q)-\xi^{(0)}(\bar{q})\right)\right] \\
& =\int d Q\left(\mu_{0}^{(0)}\left(e_{0} ; Q ; \emptyset ; \emptyset\right) f_{0}, \mu_{0}^{(0)}\left(e_{0} ; Q ; \emptyset ; \emptyset\right) f_{0}\right)_{\mathbb{C}^{2}} \\
& \times\left\{\delta\left(\bar{q}-\bar{q}^{\prime}\right)+\left[i \pi\left(A_{0}\left(\xi^{(0)}(\bar{q}) ; \bar{q}^{\prime}, \bar{q}\right)-\overline{A_{0}\left(\xi^{(0)}\left(\bar{q}^{\prime}\right) ; \bar{q}, \bar{q}^{\prime}\right)}\right)\right.\right. \\
& \left.\left.+\pi^{2} \int_{\left\{\xi^{(0)}(q)=\xi^{(0)}(\bar{q})\right\}} d q A_{0}\left(\xi^{(0)}(\bar{q}) ; q, \bar{q}\right) A_{0}\left(\xi^{(0)}\left(\bar{q}^{\prime}\right) ; q, \bar{q}^{\prime}\right)\right] \delta\left(\xi^{(0)}(\bar{q})-\xi^{(0)}\left(\bar{q}^{\prime}\right)\right)\right\}
\end{aligned}
$$

In the equality above we used Eq. (2.20). Let us remark that the vector

$$
F_{0}(Q)=\left\{\begin{array}{rr}
f_{0}, & Q=\emptyset \\
\mu_{0}^{(0)}\left(e_{0}, Q, \emptyset, \emptyset\right) f_{0}, & |Q|>0
\end{array}\right.
$$

is nothing but the eigenvector (found above) of $H$ corresponding to $e_{0}$. Hence,

$$
\begin{gathered}
\int d Q\left(\mu_{0}^{(0)}\left(e_{0} ; Q ; \emptyset ; \emptyset\right) f_{0}, \mu_{0}^{(0)}\left(e_{0} ; Q ; \emptyset ; \emptyset\right) f_{0}\right)_{\mathbb{C}^{2}} \\
=\left\|F_{0}\right\|^{2}-\left\|f_{0}\right\|^{2}=\left\|F_{0}\right\|^{2}-1=R=O\left(\alpha^{2}\right) .
\end{gathered}
$$

Collecting the expressions above, we obtain that $Q^{0,0}\left(\bar{q}, \bar{q}^{\prime}\right)$ equals

$$
\begin{aligned}
Q^{0,0}\left(\bar{q}, \bar{q}^{\prime}\right)=\left\|F_{0}\right\|^{2} & {\left[\delta\left(\bar{q}-\bar{q}^{\prime}\right)\right.} \\
& +\left\{i \pi\left(A_{0}\left(\xi^{(0)}(\bar{q}) ; \bar{q}^{\prime}, \bar{q}\right)-\overline{A_{0}\left(\xi^{(0)}\left(\bar{q}^{\prime}\right) ; \bar{q}, \bar{q}^{\prime}\right)}\right)\right. \\
& \left.+\pi^{2} \int_{\left\{\xi^{(0)}(q)=\xi^{(0)}(\bar{q})\right\}} d q A_{0}\left(\xi^{(0)}(\bar{q}) ; q, \bar{q}\right) \overline{A_{0}\left(\xi^{(0)}\left(\bar{q}^{\prime}\right) ; q, \bar{q}^{\prime}\right)}\right\} \\
& \left.\times \delta\left(\xi^{(0)}\left(\bar{q}^{\prime}\right)-\xi^{(0)}(\bar{q})\right)\right]
\end{aligned}
$$

- $i=i^{\prime}=1$. A similar calculation gives

$$
\begin{aligned}
Q^{1,1}\left(\bar{q}, \bar{q}^{\prime}\right)=\left\|F_{1}\right\|^{2} & {\left[\delta\left(\bar{q}-\bar{q}^{\prime}\right)\right.} \\
& +\left\{i \pi\left(A_{1}\left(\xi^{(1)}(\bar{q}) ; \bar{q}^{\prime}, \bar{q}\right)-\overline{A_{1}\left(\xi^{(1)}\left(\bar{q}^{\prime}\right) ; \bar{q}, \bar{q}^{\prime}\right)}\right)\right. \\
& \left.+\pi^{2} \int_{\left\{\xi^{(1)}(q)=\xi^{(1)}(\bar{q})\right\}} d q A_{1}\left(\xi^{(1)}(\bar{q}) ; q, \bar{q}\right) \overline{A_{1}\left(\xi^{(1)}\left(\bar{q}^{\prime}\right) ; q, \bar{q}^{\prime}\right)}\right\} \\
& \left.\times \delta\left(\xi^{(1)}\left(\bar{q}^{\prime}\right)-\xi^{(1)}(\bar{q})\right)\right],
\end{aligned}
$$

where $F_{0}$ and $F_{1}$ are the eigenvectors of $H$ constructed above, corresponding to the eigenvalues $e_{0}$ and $e_{1}$, respectively. 
- $i \neq i^{\prime}$. Finally,

$$
Q^{0,1}\left(\bar{q}, \bar{q}^{\prime}\right)=Q^{1,0}\left(\bar{q}^{\prime}, \bar{q}\right)=0,
$$

as these functions have as factors $\left(F_{0}, F_{1}\right)_{\mathcal{H}}=0$.

We have thus proved:

Lemma 4.3 The subspaces $\mathcal{H}^{(1)}$ and $\mathcal{H}^{(2)}$ are orthogonal. The scalar product in each of them has the form

$$
\begin{aligned}
\left(F_{\varphi_{1}}^{(i)}, F_{\varphi_{2}}^{(i)}\right)_{\mathcal{H}^{(i)}} & =\left\|F_{i}\right\|^{2} \int_{\kappa+e_{i}}^{\lambda_{0,2}^{0}-\eta} d x\left[\int_{\chi_{x}^{(i)}} \varphi_{1}(q) \overline{\varphi_{2}(q)} d \nu_{x}^{(i)}(q)\right. \\
& \left.+\int_{\chi_{x}^{(i)}} \int_{\chi_{x}^{(i)}} M_{x}^{(i)}\left(q, q^{\prime}\right) \varphi_{1}(q) \overline{\varphi_{2}\left(q^{\prime}\right)} d \nu_{x}^{(i)}(q) d \nu_{x}^{(i)}\left(q^{\prime}\right)\right], \quad i=0,1 .
\end{aligned}
$$

Here, $\chi_{x}^{(i)}$ is the level surface of the function $\xi^{(i)}(\cdot)$ :

$$
\chi_{x}^{(i)}=\left\{q: \xi^{(i)}(q)=x\right\}, \quad x \in\left[x+e_{1}, \lambda_{0,2}^{0}-\eta\right),
$$

endowed with the Gelfand-Leray measure $d \nu_{x}^{(i)}$ generated by the function $\xi^{(i)}(\cdot)$, and $M_{x}^{(i)}\left(\bar{q}, \bar{q}^{\prime}\right)$ denotes the restriction to $\chi_{x}^{(i)} \times \chi_{x}^{(i)}$ of the function

$$
\begin{aligned}
M^{(i)}\left(\bar{q}, \bar{q}^{\prime}\right) & =i \pi\left(A_{i}\left(\xi^{(i)}(\bar{q}) ; \bar{q}^{\prime}, \bar{q}\right)-\overline{A_{i}\left(\xi^{(i)}\left(\bar{q}^{\prime}\right) ; \bar{q}, \bar{q}^{\prime}\right)}\right) \\
& +\pi^{2} \int_{\left\{\xi^{(i)}(q)=\xi^{(i)}(\bar{q})\right\}} d q A_{i}\left(\xi^{(i)}(\bar{q}) ; q, \bar{q}\right) \overline{A_{i}\left(\xi^{(i)}\left(\bar{q}^{\prime}\right) ; q, \bar{q}^{\prime}\right)} .
\end{aligned}
$$

Our estimates imply that the operators $M^{(i)}, i=0,1$ given by the kernels $M^{(i)}\left(\bar{q}, \bar{q}^{\prime}\right), i=$ 0,1 are bounded in $\mathcal{H}^{(i)}, i=0,1$, respectively, and their norms are $\leq 1$ for small $\alpha$. This, and the formulas (4.32), (4.33), imply, in particular, that

$$
C_{0}\|\varphi\|_{L_{2}\left(G_{\eta}^{(i)}\right)}<\left\|F_{\varphi}^{(i)}\right\|_{\mathcal{H}^{(i)}}<C_{1}\|\varphi\|_{L_{2}\left(G_{\eta}^{(i)}\right)}
$$

for certain constants $0<C_{0}<C_{1}$, therefore the applications $\varphi \mapsto F_{\varphi}^{(i)}: \mathcal{C}^{(i)} \rightarrow \mathcal{H}^{(i)}$ are continuous with respect to the $L_{2}\left(G_{\eta}^{(i)}\right)$-norm, and, as such, they extend to one-toone applications: $L_{2}\left(G_{\eta}^{(i)}\right) \rightarrow \mathcal{H}^{(i)}, \varphi \mapsto F_{\varphi}^{(i)}, \varphi \in L_{2}\left(G_{\eta}^{(i)}\right)$. Thereby, the action of the operator $H$ on a vector $F_{\varphi}^{(i)} \in \mathcal{H}^{(i)}$ is given by the formula

$$
H F_{\varphi}^{(i)}=F_{\xi^{(i)} \varphi}^{(i)},
$$

where $\left(\xi^{(i)} \varphi\right)(q)=\xi^{(i)}(q) \varphi(q), q \in G_{\eta}^{(i)}$.

As seen from Eq. (4.33), each of the spaces $\mathcal{H}^{(i)}$ can be represented as a direct integral of spaces

$$
\mathcal{H}^{(i)}=\int_{\left[e_{i}+\kappa, \lambda_{0,2}^{0}-\eta\right]}^{\oplus} \mathcal{G}_{x}^{(i)} d x \text {, where } \mathcal{G}_{x}^{(i)}=L_{2}\left(\chi_{x}^{(i)}, d \nu_{x}^{(i)}\right),
$$


whereby the spaces $\mathcal{G}_{x}^{(i)}$ are "eigenspaces" of $H^{(i)}=\left.H\right|_{\mathcal{H}^{(i)}}$, i.e.

$$
H^{(i)}=\int_{\left[e_{i}+\kappa, \lambda_{0,2}^{0}-\eta\right]}^{\oplus} x I_{x}^{(i)} d x
$$

where $I_{x}^{(i)}$ is the unit operator in $\mathcal{G}_{x}^{(i)}$.

Lemma 4.4 For each $i=0,1$ there exists a bounded operator $B^{(i)}$ acting in $\mathcal{H}^{(i)}$ and commuting with $H^{(i)}$, such that

$$
\left(F_{B^{(i)} \varphi_{1}}^{(i)}, F_{B^{(i)} \varphi_{2}}^{(i)}\right)=\left(\varphi_{1}, \varphi_{2}\right)_{L_{2}\left(G_{\eta}^{(i)}\right)}, \varphi_{1}, \varphi_{2} \in L_{2}\left(G_{\eta}^{(i)}\right)
$$

Proof. The scalar product (4.33) induces in $\mathcal{G}_{x}^{(i)}$ a sesquilinear form:

$$
\left\langle\varphi_{1}, \varphi_{2}\right\rangle_{\mathcal{G}_{x}^{(i)}}=\left\|F^{(i)}\right\|_{2}\left(\left(I_{x}^{(i)}+M_{x}^{(i)}\right) \varphi_{1}, \varphi_{2}\right)_{\mathcal{G}_{x}^{(i)}}
$$

where $\left(\varphi_{1}, \varphi_{2}\right)_{\mathcal{G}_{x}^{(i)}}$ is the scalar product in $\mathcal{G}_{x}^{(i)}$ and $M_{x}^{(i)}$ is the selfadjoint operator in $\mathcal{G}_{x}^{(i)}$ defined by the kernel $M_{x}^{(i)}\left(\bar{q}, \bar{q}^{\prime}\right)$. Our estimates show that $\left\|M_{x}^{(i)}\right\|<1$ for $\alpha$ small, hence that the bounded operator $B_{x}^{(i)}=\left(I_{x}^{(i)}+M_{x}^{(i)}\right)^{-1 / 2}$ exists. Obviously,

$$
\left\langle B_{x}^{(i)} \varphi_{1}, B_{x}^{(i)} \varphi_{2}\right\rangle_{\mathcal{G}_{x}^{(i)}}=\left(\varphi_{1}, \varphi_{2}\right)_{\mathcal{G}_{x}^{(i)}}
$$

Therefore, the operator $B^{(i)}=\int_{\left[e_{i}+\kappa, \lambda_{0,2}^{0}-\eta\right]}^{\oplus} B_{x}^{(i)} d x$, which commutes with $H^{(i)}$, satisfies the condition (4.36) as well. The lemma is proved.

The Lemma 4.4 shows that the application $\varphi \mapsto \hat{F}_{\varphi}: L_{2}\left(G_{\eta}^{(i)}\right) \rightarrow \mathcal{H}^{(i)}$, where $\hat{F}_{\varphi}=$ $F_{B^{(i)} \varphi}, \varphi \in L_{2}\left(G_{\eta}^{(i)}\right)$, is unitary. Thereby, as $B^{(i)}$ commutes with $H^{(i)}$, the relation (4.35) still holds:

$$
H \hat{F}_{\varphi}^{(i)}=\hat{F}_{\xi^{(i)}}^{(i)}
$$

\section{Conclusion}

In conclusion we would like to notice that observations made in Sections 3 and 4 can be resumed as the following statement:

Theorem 5.1 Under the assumptions made concerning the parameters of the model (1.1), and for a sufficiently small, for the operator $H$ one has:

(i) One (or two) eigenvectors $F_{0}^{(i)}$ with the eigenvalues $e_{i}$, respectively, below its continuous spectrum $(i=0$, or $i=0,1)$.

(ii) For any $\eta$, one, or two (depending on the number of eigenvectors $F_{0}^{(i)}$ ), invariant 
subspaces $\mathcal{H}^{(i)}$, and one, or two, bounded domains $G_{\eta}^{(i)} \subset \mathbb{R}^{d}$, such that the restriction of $H$ to $\mathcal{H}^{(i)}$ is unitarily equivalent to the operator of multiplication by the function

$$
\xi^{(i)}(q)=e_{i}+\omega(q), q \in G_{\eta}^{(i)}
$$

acting in $L_{2}\left(G_{\eta}^{(i)}\right)$.

This theorem summarizes our main result about the structure of the spectrum of a twolevel quantum system weakly coupled to a boson field (spin-boson model) announced in the Section 1 .

In conclusion we would like to note that (in spite of the technical difficulties) we believe that our method allows some generalizations and improvements to be able:

(i) to construct in a similar way the multi-boson branches; cf. [6], where it is nicely done in a different way (a limited space of the present paper does not allow us to enter into details of [6] and we recommend it for the reader as an important reference);

(ii) to prove the completeness, which reduces to the proof that the Hilbert space $\mathcal{H}=$ $\mathbb{C}^{2} \otimes \mathcal{F}_{s}$ in imbedded by a nuclear operator into the space $\tilde{\tilde{\mathcal{B}}}^{\prime}$, see Remark 2.5 and (2.26).

A project concerning these two points is now in progress.

Acknowledgements. N. A. and R. A. M. thank Centre de Physique Théorique (UMR 6207) - Luminy, where this work was initiated, for financial support and the warm hospitality. N.A. acknowledges the financial support of the CERES and CEEX programs (Grants No. 4-187/2004 and 05-D11-06). R. A. M. acknowledges the financial support from the funds RFFI and CRDF.

We would like to thank referees for very relevant remarks and constructive criticism. They motivated us to take a time to revise and to make more precise the presentation of results that we are able to proof at the present time. 


\section{Appendix}

Proof of Proposition 3.1:

Let $\mathcal{K}_{1}$ be a space of smooth functions: $f(q, k) \in \mathcal{M}_{2}$ for $q, k \in \mathbb{R}^{d}$, such that

$$
\left\|\left(\partial_{q}^{\varepsilon_{1}} \partial_{k}^{\varepsilon_{2}} f\right)(q, k)\right\| \leq C h(q) h(k), \quad \varepsilon_{1,2}=0,1 .
$$

Here $\partial_{q}^{\varepsilon}:=\left(\partial_{q_{1}}^{\varepsilon}, \ldots, \partial_{q_{d}}^{\varepsilon}\right)$ and $\|\cdot\|$ is equal to the sum of the matrix-norms of $\left\{\partial_{q_{i}}^{\varepsilon_{1}} \partial_{q_{j}}^{\varepsilon_{2}} f\right\}_{i, j=1, \varepsilon_{1}, \varepsilon_{2}}^{d}$. We define the norm in $\mathcal{K}_{1}$ by

$$
\|f\|_{\mathcal{K}_{1}}:=\inf C
$$

over $C$ verifying (6.1).

Recall that the kernels $\left\{\hat{D}_{1,1}(z ; q ; \emptyset ; k)\right\}_{z}$ belong to the family of $\mathcal{K}_{1}$-valued functions $\mathcal{D}_{z}(q, k)$ defined in the semi-plane

$$
D_{1, \eta}=\left\{z \in \mathbb{C}: \operatorname{Re} z<\lambda_{0,2}^{0}-\eta=2 \kappa-\varepsilon-\eta\right\},
$$

see (2.12), with the cut along the interval (see Proposition 3.11):

$$
I=\left(\kappa+e_{0}, 2 \kappa-\varepsilon-\eta\right)
$$

We assume that:

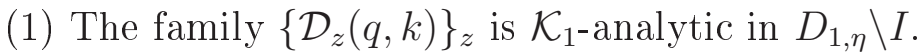

(2) It is also $\mathcal{K}_{1}$-continuous and bounded: $\left\|\mathcal{D}_{z}\right\|_{\mathcal{K}_{1}}<L_{1}$, in the closure $\overline{D_{1, \eta} \backslash I}$.

(3) $\lim _{z \rightarrow 0}\left\|\mathcal{D}_{z}\right\|_{\mathcal{K}_{1}}=0$.

(4) For $x \in I$ the limit values $\mathcal{D}_{x}^{ \pm}(q, k):=\lim _{z \rightarrow \pm x} \mathcal{D}_{z}(q, k)$ verify the Hölder condition:

$$
\left|\mathcal{D}_{x_{1}}^{ \pm}(\cdot, \cdot)-\mathcal{D}_{x_{2}}^{ \pm}(\cdot, \cdot)\right|<C_{1}\left|x_{1}-x_{2}\right|^{1 / 2}
$$

for constant $C_{1}$ and exponent $1 / 2$.

By (3.6) the kernel of the inverse operator $B(z)^{-1}$ has the form:

$$
\begin{aligned}
{\left[B(z)^{-1}\right](q, k) } & =\left[\varepsilon \sigma_{3}-(z-\omega(q))-m(z-\omega(q))\right]^{-1} \\
& \times\left\{\delta(q, k)+K(z ; q, k)\left[\varepsilon \sigma_{3}-(z-\omega(k))-m(z-\omega(k))\right]^{-1}\right\}
\end{aligned}
$$

where (formally) we put:

$$
K(z ; q, k)=\sum_{n=1}^{\infty} K^{(n)}(z ; q, k)
$$

with $K^{(n=1)}(z ; q, k):=\mathcal{D}_{z}(q, k)$ and the other terms are defined by recursions

$$
K^{(n+1)}(z ; q, k):=\int_{\mathbb{R}^{d}} K^{(n)}\left(z ; q, q^{\prime}\right)\left[\varepsilon \sigma_{3}-\left(z-\omega\left(q^{\prime}\right)\right)-m\left(z-\omega\left(q^{\prime}\right)\right)\right]^{-1} K^{(1)}\left(z ; q^{\prime}, k\right) d q^{\prime}
$$

for $n+1 \geq 2$. 
Let for a given $n$ the kernel $K^{(n)}\left(z ; q, q^{\prime}\right)$ verifies the same conditions (1)-(4) as the function $\mathcal{D}_{z}(q, k)$, we denote them $(1)^{(n)}-(4)^{(n)}$ with the evident substitutions of the corresponding constants by $L_{n}$ and $C_{n}$. Now we have to prove that the function $K^{(n+1)}\left(z ; q, q^{\prime}\right)$ satisfies conditions $(1)^{(n+1)}-(4)^{(n+1)}$ with constants that verify the estimates:

$$
L_{n+1}<\theta L_{n} \quad \text { and } \quad C_{n+1}<\theta C_{n},
$$

for some $0<\theta<1$. Notice that (6.9) implies (a uniform in the closure $\overline{D_{1, \eta} \backslash I}$ ) convergence of (6.7) in the $\mathcal{K}_{1}$-norm, i.e. the the kernel $K\left(z ; q, q^{\prime}\right)$ verifies the conditions (1)-(4) for some $L_{\infty}$ and $C_{\infty}$.

To this end we first use the representation:

$$
\left[\varepsilon \sigma_{3}-\left(z-\omega\left(q^{\prime}\right)\right)-m\left(z-\omega\left(q^{\prime}\right)\right)\right]^{-1}=\frac{A_{0}}{e_{0}-z-\omega\left(q^{\prime}\right)}+\frac{A_{1}}{e_{1}-z-\omega\left(q^{\prime}\right)}+\varphi\left(z-\omega\left(q^{\prime}\right)\right) .
$$

Here $A_{0}$ and $A_{1}$ are two residues of the matrix-valued rational complex function $\Phi(\zeta):=$ $\left[\varepsilon \sigma_{3}-\zeta-m(\zeta)\right]^{-1}$ at poles $e_{0}$ and $e_{1}$ respectively, see (3.2), and $\varphi: \mathbb{C} \mapsto \mathcal{M}_{2}$ is an analytic function bounded in domain $\left\{\zeta \in \mathbb{C}: \operatorname{Re} \zeta<\lambda_{0,1}^{0}-\eta=\kappa-\varepsilon-\eta\right\}$. Then (6.8) yields:

$$
K^{(n+1)}(z ; q, k)=K^{(n+1), 0}(z ; q, k)+K^{(n+1), 1}(z ; q, k)+\widehat{K}^{(n+1)}(z ; q, k),
$$

where $(j=0,1)$

$$
\begin{aligned}
& K^{(n+1), j}(z ; q, k):=\int_{\mathbb{R}^{d}} K^{(n)}\left(z ; q, q^{\prime}\right) \frac{A_{j}}{e_{j}-z-\omega\left(q^{\prime}\right)} K^{(1)}\left(z ; q^{\prime}, k\right) d q^{\prime} \\
& \widehat{K}^{(n+1)}(z ; q, k):=\int_{\mathbb{R}^{d}} K^{(n)}\left(z ; q, q^{\prime}\right) \varphi\left(z-\omega\left(q^{\prime}\right)\right) K^{(1)}\left(z ; q^{\prime}, k\right) d q^{\prime}
\end{aligned}
$$

The recursions (6.12) and (6.13) imply $\mathcal{K}_{1}$-analyticity of terms (6.11) in domain $D_{1, \eta} \backslash I$ and the estimates:

$$
\begin{aligned}
& \max \left\{\left\|\partial_{z} K^{(n+1), j}(z ; q, k)\right\|_{\mathcal{K}_{1}} ;\left\|K^{(n+1), j}(z ; q, k)\right\|_{\mathcal{K}_{1}}\right\}<\text { Const } \times \\
& \max \left\{\left\|\partial_{z} K^{(n), j}(z ; q, k)\right\|_{\mathcal{K}_{1}} ;\left\|K^{(n), j}(z ; q, k)\right\|_{\mathcal{K}_{1}}\right\} \max \left\{\left\|\partial_{z} K^{(1), j}(z ; q, k)\right\|_{\mathcal{K}_{1}} ;\left\|K^{(1), j}(z ; q, k)\right\|_{\mathcal{K}_{1}}\right\} \times \\
& \left\{\frac{1}{(\operatorname{dist}(z, I))^{2}}+\frac{1}{\operatorname{dist}(z, I)}\right\},
\end{aligned}
$$

where $\operatorname{dist}(z, I)$ is the distance between $z$ and the cut $I$. Similarly one also obtains:

$$
\begin{aligned}
& \max \left\{\left\|\partial_{z} \widehat{K}^{(n+1)}(z ; q, k)\right\|_{\mathcal{K}_{1}} ;\left\|\widehat{K}^{(n+1)}(z ; q, k)\right\|_{\mathcal{K}_{1}}\right\}<\text { Const } \times \\
& \max _{\operatorname{map}}\left\{\left\|\partial_{z} \widehat{K}^{(n)}(z ; q, k)\right\|_{\mathcal{K}_{1}} ;\left\|\widehat{K}^{(n)}(z ; q, k)\right\|_{\mathcal{K}_{1}}\right\} \max \left\{\left\|\partial_{z} \widehat{K}^{(1)}(z ; q, k)\right\|_{\mathcal{K}_{1}} ;\left\|\widehat{K}^{(1)}(z ; q, k)\right\|_{\mathcal{K}_{1}}\right\} \times \\
& \zeta \in\left(D_{1, \eta \backslash I)}\right.
\end{aligned}
$$

Moreover, for any $n$ the function $\widehat{K}^{(n)}(z ; q, k)$ is $\mathcal{K}_{1}$-continuous on the closure $\overline{D_{1, \eta} \backslash I}$ and one gets for the limit values on the cut:

$$
\begin{aligned}
& \widehat{K}^{(n+1), \pm}(x ; q, k):=\lim _{z \rightarrow \pm x} \widehat{K}^{(n+1)}(z ; q, k)= \\
& \int_{\mathbb{R}^{d}} K^{(n), \pm}\left(x ; q, q^{\prime}\right) \varphi\left(x-\omega\left(q^{\prime}\right)\right) K^{(1), \pm}\left(x ; q^{\prime}, k\right) d q^{\prime} .
\end{aligned}
$$


Then estimate (6.5), condition $(4)^{(n)}$, and (6.14) yield

$$
\left\|\widehat{K}^{(n+1), \pm}\left(x_{1} ; \cdot, \cdot\right)-\widehat{K}^{(n+1), \pm}\left(x_{2} ; \cdot, \cdot\right)\right\|_{\mathcal{K}_{1}}<R_{n+1}\left|x_{1}-x_{2}\right|^{1 / 2}
$$

where

$$
\begin{aligned}
R_{n+1}= & \text { Const } \max \left\{C_{n}, \sup _{x \in I}\left\|K^{(n), \pm}(x ; \cdot, \cdot)\right\|_{\mathcal{K}_{1}}\right\} \times \\
& \max \left\{C_{1}, \sup _{x \in I}\left\|K^{(1), \pm}(x ; \cdot, \cdot)\right\|_{\mathcal{K}_{1}}\right\} \underset{\zeta \in\left(\overline{D_{1, \eta} \backslash I}\right)}{\max }|\varphi(\zeta)| .
\end{aligned}
$$

By virtue of (6.14) one also gets that $\lim _{z \rightarrow \infty}\left\|\widehat{K}^{(n+1)}(z ; \cdot, \cdot)\right\|=0$. Therefore, the family $\left\{\widehat{K}^{(n+1)}(z ; \cdot, \cdot)\right\}_{z}$ verifies the conditions $(1)^{(n+1)}-(4)^{(n+1)}$.

Now we have to check the same properties for the kernel $K^{(n+1)}(z ; \cdot, \cdot)$. To this end we introduce

$$
B(z ; q, k \mid y>\kappa):=\int_{\Gamma_{y}:=\left\{q^{\prime}: \omega\left(q^{\prime}\right)=y\right\}} K^{(n)}\left(z ; q, q^{\prime}\right) K^{(1)}\left(z ; q^{\prime}, q\right) d \nu_{y}\left(q^{\prime}\right),
$$

with integration over the Gelfand-Leray measure $\nu_{y}(\cdot)$ on the surface $\Gamma_{y}$, see [3]. Notice that by (6.16) one gets:

$$
|B(z ; q, k \mid y)|<\left\|K^{(n)}(z ; \cdot, \cdot)\right\|_{\mathcal{K}_{1}}\left\|K^{(1)}(z ; \cdot, \cdot)\right\|_{\mathcal{K}_{1}} h(q) h(k) \int_{\Gamma_{y}} h\left(q^{\prime}\right)^{2} d \nu_{y}\left(q^{\prime}\right) .
$$

Thus, $B \in \mathcal{K}_{1}$ for any $z \in \overline{D_{1, \eta} \backslash I}$ and $y>\kappa$ with the norm-estimate:

$$
\|B(z ; \cdot, \cdot \mid y)\|_{\mathcal{K}_{1}}<\left\|K^{(n)}(z ; \cdot, \cdot)\right\|_{\mathcal{K}_{1}}\left\|K^{(1)}(z ; \cdot, \cdot)\right\|_{\mathcal{K}_{1}} \int_{\Gamma_{y}} h\left(q^{\prime}\right)^{2} d \nu_{y}\left(q^{\prime}\right),
$$

and

$$
\int_{0}^{\infty}\|B(z ; \cdot, \cdot \mid y)\|_{\mathcal{K}_{1}} d y<\left\|K^{(n)}(z ; \cdot, \cdot)\right\|_{\mathcal{K}_{1}}\left\|K^{(1)}(z ; \cdot, \cdot)\right\|_{\mathcal{K}_{1}} \int_{\mathbb{R}^{d}} h\left(q^{\prime}\right)^{2} d q^{\prime}
$$

Now we can prove the following estimates of $y$-derivative of $B$ :

$$
\left\|\partial_{y} B(z ; \cdot, \cdot \mid y)\right\|_{\mathcal{K}_{1}}<C(y)\left\|K^{(n)}(z ; \cdot, \cdot)\right\|_{\mathcal{K}_{1}}\left\|K^{(1)}(z ; \cdot, \cdot)\right\|_{\mathcal{K}_{1}}
$$

where the asymptotic of the function $C(y)$ for $y \searrow \kappa$ is

$$
C(y)=(y-\kappa)^{(d / 2-2)}+\mathcal{O}\left((y-\kappa)^{(d / 2-2)-\epsilon}\right), \quad \epsilon>0,
$$

whereas out of this $\kappa$-vicinity the function $C(y)$ is bounded.

Lemma 6.1 Let $f(q)$ be a smooth function on $\mathbb{R}^{d}$. Let us define the function

$$
\mathcal{I}(y):=\int_{\Gamma_{y}} f(q) d \nu_{y}(q) .
$$

Then we have

$$
\left|\partial_{y} \mathcal{I}(y)\right|<d^{2} \int_{\Gamma_{y}}\left\{\frac{|(\nabla f)(q)|}{|(\nabla \omega)(q)|}+|f(q)| \frac{|(\Delta \omega)(q)|}{|(\nabla \omega)(q)|^{2}}\right\} d \nu_{y}(q) .
$$


Proof. Notice that using differential forms [9] one can rewrite (6.22) as

$$
\mathcal{I}(y):=\int_{\Gamma_{y}} \Omega_{0}(f)
$$

where $\Omega_{0}(f)=f \widetilde{\Omega}$ and $\widetilde{\Omega}$ is a special (Gelfand-Leray form), which has the following local coordinate representation:

$$
\widetilde{\Omega}=\frac{1}{\partial \omega / \partial q_{1}} d q_{2} \ldots d q_{d}
$$

Without lost of generality we can suppose that $\partial \omega / \partial q_{1} \neq O$ and $\left|\partial \omega / \partial q_{1}\right| \geq\left|\partial \omega / \partial q_{j}\right|, j=$ $2, \ldots, d$. By (6.24) and (6.25) one gets [9]:

$$
\partial_{y} \mathcal{I}(y)=\int_{\Gamma_{y}} \Omega_{1}(f), \quad \Omega_{1}(f)=\left\{\partial_{q_{1}} f \frac{1}{\partial \omega / \partial q_{1}}+f \partial_{q_{1}} \frac{1}{\partial \omega / \partial q_{1}}\right\} \widetilde{\Omega} .
$$

Since the convexity of $\omega$ implies $d\left|\partial \omega / \partial q_{1}\right| \geq|\nabla \omega|$ and $\left|\partial^{2} \omega / \partial q_{1}^{2}\right|<|\Delta \omega|$, we obtain the estimate (6.23).

Corollary 6.2 The estimate (6.23) implies (6.20) with

$$
\begin{aligned}
C(y) & =d^{2} \int_{\Gamma_{y}}\left\{\frac{2 h(q)^{2}}{|(\nabla \omega)(q)|}+h(q)^{2} \frac{|(\Delta \omega)(q)|}{|(\nabla \omega)(q)|^{2}}\right\} d \nu_{y}(q)= \\
& =d^{2} \partial_{y} \int_{\{\kappa<\omega(q)<y\}} h(q)^{2}\left\{\frac{2}{|(\nabla \omega)(q)|}+\frac{|(\Delta \omega)(q)|}{|(\nabla \omega)(q)|^{2}}\right\} d q .
\end{aligned}
$$

Moreover, since for $y \searrow \kappa$ one gets in domain of integration: $|(\nabla \omega)(q)| \sim \sqrt{y-\kappa}$ and $d q \sim|y-\kappa|^{d / 2-1} d y$, the right-hand side of the last identity in (6.27) has asymptotics $(y-\kappa)^{(d / 2-2)}$ that proves (6.21). On the other hand, for $y>\kappa+\epsilon, \epsilon \geq \epsilon_{0}>0$ the same expression is bounded.

By virtue of (6.20) and (6.21) we get for $d=3$ the $B(z ; \cdot, \cdot \mid y)$ verifies the Hölder condition with exponent $1 / 2$ :

$$
\left\|B\left(z ; \cdot, \cdot \mid y_{1}\right)-B\left(z ; \cdot, \cdot \mid y_{2}\right)\right\|_{\mathcal{K}_{1}}<\widehat{C}_{3}\left|y_{1}-y_{2}\right|^{1 / 2}
$$

whereas for $d>3$ we obtain:

$$
\left\|B\left(z ; \cdot, \cdot \mid y_{1}\right)-B\left(z ; \cdot, \cdot \mid y_{2}\right)\right\|_{\mathcal{K}_{1}}<\widehat{C}_{>3}\left|y_{1}-y_{2}\right| .
$$

Finally, $B(z ; \cdot, \cdot \mid y)$ is continuous in the closure $\overline{D_{1, \eta} \backslash I}$, as a function of $z$, and by (6.16) we have for it on the cut $I$ the values:

$$
B^{ \pm}(x ; q, k \mid y):=\int_{\Gamma_{y}} K^{(n), \pm}\left(x ; q, q^{\prime}\right) K^{(1), \pm}\left(x ; q^{\prime}, q\right) d \nu_{y}\left(q^{\prime}\right),
$$

which satisfy the above estimates (6.18)-(6.20) and (6.28) or (6.29), for $z=x$. 
Moreover, we find behaviour of (6.30) as a function of $x$ :

$$
\left\|B\left(x_{1} ; \cdot, \cdot \mid y\right)-B\left(x_{2} ; \cdot, \cdot \mid y\right)\right\|_{\mathcal{K}_{1}}<\tilde{C}_{d}\left|x_{1}-x_{2}\right|^{\gamma_{d}}
$$

where $\tilde{C}_{d}=L_{n} C_{1}+L_{1} C_{n}\left(\right.$ see $(\underline{6.9})$ ) and $\gamma_{d=3}=1 / 2, \gamma_{d>3}=1$.

Summarizing we conclude that for $j=0$ we obtain the corresponding representation of the kernel (6.12) in the form:

$$
K^{(n+1), 0}(z ; q, k)=\int_{\kappa}^{\infty} \frac{B(z ; q, k \mid y)}{e_{0}+y-z} d y .
$$

Now to establish desired properties, $(1)^{(n+1)}-(4)^{(n+1)}$, of this kernel on the basis of the $B(z ; q, k \mid y)$ properties, we consider a more general integral:

$$
\widetilde{K}^{(n+1), 0}\left(z_{1}, z_{2} ; q, k\right)=\int_{\kappa}^{\infty} \frac{B\left(z_{1} ; q, k \mid y\right)}{e_{0}+y-z_{2}} d y .
$$

Lemma 6.3 (Privalov's lemma for vector-valued functions)

Let $\{f(z, y)\}_{z, y} \subset \mathcal{B}$ for $z \in \mathcal{D}_{0} \backslash I$ with a cut $I$ and $y \in(\kappa, \infty)$ be family of vector-valued functions in a Banach space $\mathcal{B}$. Assume that they verify the following conditions:

(a) $f(z, y)$ is $\mathcal{B}$-analytic in $\mathcal{D}_{0} \backslash I$ and $\mathcal{B}$-continuous on the closure $\overline{\mathcal{D}_{0} \backslash I}$ for any fixed $y \in(\kappa, \infty)$.

(b) The limit values $f^{ \pm}(x, y)$ on the cut I verify the Hölder condition:

$$
\left\|f^{ \pm}\left(x_{1}, y\right)-f^{ \pm}\left(x_{2}, y\right)\right\|_{\mathcal{B}}<C_{1}\left|x_{1}-x_{2}\right|^{1 / 2}, x_{1,2} \in I
$$

(c) For any $z \in \overline{\mathcal{D}_{0} \backslash I}$ one has

$$
\left\|f\left(z, y_{1}\right)-f\left(z, y_{2}\right)\right\|_{\mathcal{B}}<C_{2}\left|y_{1}-y_{2}\right|^{1 / 2}, y_{1,2} \in(\kappa, \infty) \text {. }
$$

(d) For any $z \in \overline{\mathcal{D}_{0} \backslash I}$ the integral

$$
\int_{\kappa}^{\infty}\|f(z, y)\|_{\mathcal{B}} d y<R
$$

(f) Uniform boundedness and limits at infinity:

$$
\sup _{\left\{z \in \overline{\mathcal{D}_{0} \backslash I}, y \in(\kappa, \infty)\right\}}\|f(z, y)\|_{\mathcal{B}}<M \text { and } \lim _{z \rightarrow \infty}\|f(z, y)\|_{\mathcal{B}}=\lim _{y \rightarrow \infty}\|f(z, y)\|_{\mathcal{B}}=0 .
$$

Let us define the (Bochner) integral

$$
F\left(z_{1}, z_{2}\right):=\int_{\kappa}^{\infty} \frac{f\left(z_{1}, y\right)}{e_{0}+y-z_{2}} d y
$$

where $e_{0}$ is defined by the cut $I$ (6.4). Then the function (6.38) has the following properties: 
(1) It is analytic in $\mathcal{D}:=\left\{\mathcal{D}_{0} \backslash I\right\} \times\left\{\mathcal{D}_{0} \backslash I\right\}$ in two variables $z_{1}, z_{2}$. (2) It is uniformly bounded and continuous in the closure $\overline{\mathcal{D}}$ :

$$
\left\|F\left(z_{1}, z_{2}\right)\right\|_{\mathcal{B}}<A_{1} M
$$

(3) The limit values $F^{ \pm}\left(x_{1}, x_{2}\right)$ on the cut I satisfy the Hölder conditions:

$$
\left\|F^{ \pm}\left(x_{1}+\delta_{1}, x_{2}+\delta_{2}\right)-F^{ \pm}\left(x_{1}, x_{2}\right)\right\|_{\mathcal{B}}<\widehat{C}\left(\left|\delta_{1}\right|^{1 / 2}+\left|\delta_{2}\right|^{1 / 2}\right)
$$

where

$$
F^{ \pm}\left(x_{1}, x_{2}\right):=\lim _{\varepsilon_{1,2} \rightarrow+0} F\left(x_{1} \pm i \varepsilon_{1}, x_{2} \pm i \varepsilon_{2}\right)
$$

and $\widehat{C}=A_{2}\left(C_{1}+C_{2}\right)$.

Proof. Follows through verbatim of the standard demonstration for complex-valued functions, see e.g. [16].

Applying Lemma 6.3 to our case of $\mathcal{B}=\mathcal{K}_{1}$ shows that limit values $\widetilde{K}^{(n+1), 0, \pm}\left(x_{1}, x_{2} ; q, k\right)$ of integral (6.33) verify the Hölder condition for variables $x_{1}, x_{2}$. Since

$$
K^{(n+1), 0}(z ; q, k)=\widetilde{K}^{(n+1), 0, \pm}(z, z ; q, k),
$$

we obtain that the function $K^{(n+1), 0, \pm}(x ; q, k)$ also verifies the Hölder condition for the variable $x$. Moreover, from the estimates that contain the factor $\max _{\left\{z \in \overline{D_{1, \eta} \backslash I}\right\}}\left\|K^{(1)}(z ; \cdot, \cdot)\right\| \|_{\mathcal{K}_{1}}=$ $\alpha$ yield:

$$
\left\|K^{(n+1), 0}(z ; \cdot, \cdot)\right\|_{\mathcal{K}_{1}}<\text { const } \alpha\left\|K^{(n)}(z ; \cdot, \cdot)\right\|_{\mathcal{K}_{1}}
$$

and

$$
\left\|K^{(n+1), 0, \pm}\left(x_{1} ; \cdot, \cdot\right)-K^{(n+1), 0, \pm}\left(x_{2} ; \cdot, \cdot\right)\right\|_{\mathcal{K}_{1}}<\text { const } \alpha C_{n}
$$

see (6.9).

Similarly one checks these estimates for the family $\left\{K^{(n+1), 1}(z ; \cdot, \cdot)\right\}_{z}$. Therefore, the whole family $\left\{K^{(n+1)}(z ; \cdot, \cdot)\right\}_{z}$ verifies the recurrent estimates (6.9) , that finishes the proof of Proposition 3.1. 


\section{References}

[1] N. Angelescu, R.A Minlos, and V.A Zagrebnov, Lower Spectral Branches of a Particle Coupled to a Bose Field, Rev.Math.Phys. 17, 1111-1142 (2005).

[2] A. Arai and M. Hirokawa, On the existence and uniqueness of ground states of a generalized spin-boson model, J.Funct.Anal. 151 , 455-503 (1997).

[3] V. Arnold, A. Varchenko, and S. Gusein-Zade, Singularities of differentiable maps, I. The classification of critical points, caustics and wave fronts. Monographs in Mathematics, 82. (Birkhäuser Boston, Inc., Boston, MA, 1985).

[4] F. A. Berezin, The method of second quantization (Academic Press, New York, 1966).

[5] O. Bratelli and D. W. Robinson, Operator Algebras and Quantum Statistical Mechanics, Vol.2 (Springer, New York, 1981).

[6] J. Dereziński, and C. Gérard, Asymptotic completeness in quantum field theory. Massive Pauli-Fierz Hamiltonians, Rev.Math.Phys. 11, 383-450 (1999).

[7] R. Dümke and H. Spohn, Quantum tunneling with dissipation and the Ising model over R, J.Statistical Phys. 41, 389-424 (1985).

[8] M. Fannes, B. Nachtergaele and A. Verbeure, The equilibrium states of the spinboson model. Comm. Math. Phys. 114, 537-548 (1988).

[9] I. M. Gelfand and G. E. Shilov, Generalized Functions, Vol. 1 (Academic Press, New York, 1964).

[10] C. Gérard, Asymptotic completeness for the spin-boson model with particle number cutoff, Rev.Math.Phys. 8, 549-589 (1996).

[11] M. Hübner and H. Spohn, Spectral properties of the spin-boson hamiltonian, Ann.Inst.H.Poincaré 62, 289-323 (1995).

[12] R.A Minlos, Lower branch of the spectrum of a fermion interacting with a bosonic gas (polaron), Teoret.Mat.Fiz. 92, 255-268 (1992).

[13] R.A Minlos, Lower branch of the spectrum of the Hamiltonian of the spinless PauliFierz model (a two-component Bose field interacting with a charged particle), Trudy Mosk. Mat. Obshch. (2006) (submitted).

[14] R.A Minlos and H. Spohn, The three-body problem in radiative decay: the case of one atom and at most two photons, Amer.Math.Soc.Translations (2) 177, 159-194 (1996).

[15] N. I. Muskhelishvili, Singular Integral Equations, (Wolters-Noordhoff, 1972). 
[16] I. I. Privalov, Boundary properties of analytic functions, 2nd ed., (GITTL, MoscowLeningrad, 1950) (in Russian);

[17] M. Reed and B. Simon, Methods of Modern Mathematical Physics, Vol.3 (Academic Press, New York, 1983).

[18] M. Reed and B. Simon, Methods of Modern Mathematical Physics, Vol.4 (Academic Press, New York, 1983).

[19] H. Spohn, Ground state(s) of the spin-boson Hamiltonian, Commun.Math.Phys. 123, 277-304 (1988).

[20] Y. V. Zhukov and R.A Minlos, Spectrum and scattering in a "spin-boson" model with not more than three photons, Teoret.Mat.Fiz. 103, 63-81 (1995). 


\section{Figures Captions}

- Figure 1a: The graph of the 1.h.s. of (3.3) : case of two roots

- Figure 1b: The graph of the 1.h.s. of (3.3) : case of one root

- Figure 2: The graph of the 1.h.s. of (4.3)

- Figure 3: Solving Eq. 4.20) 


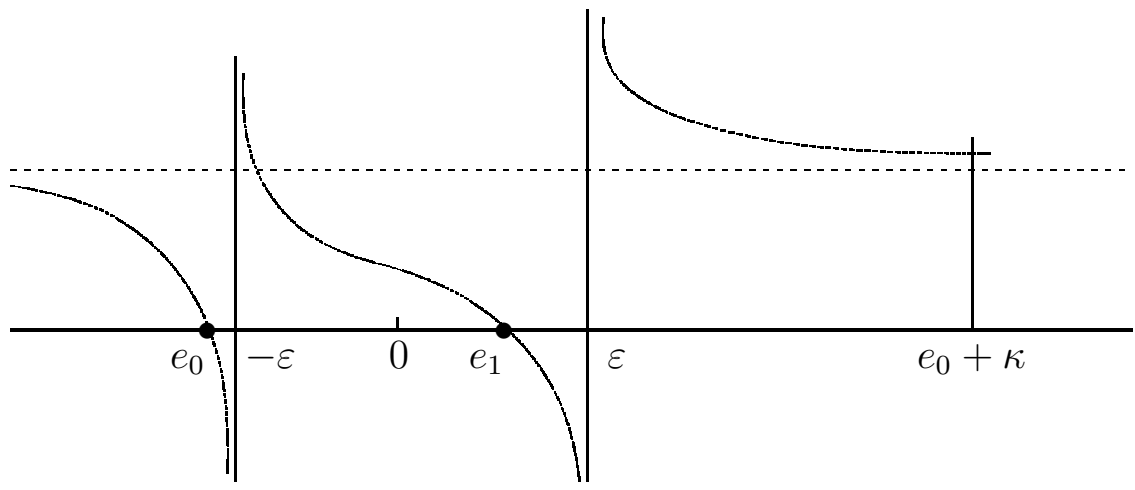




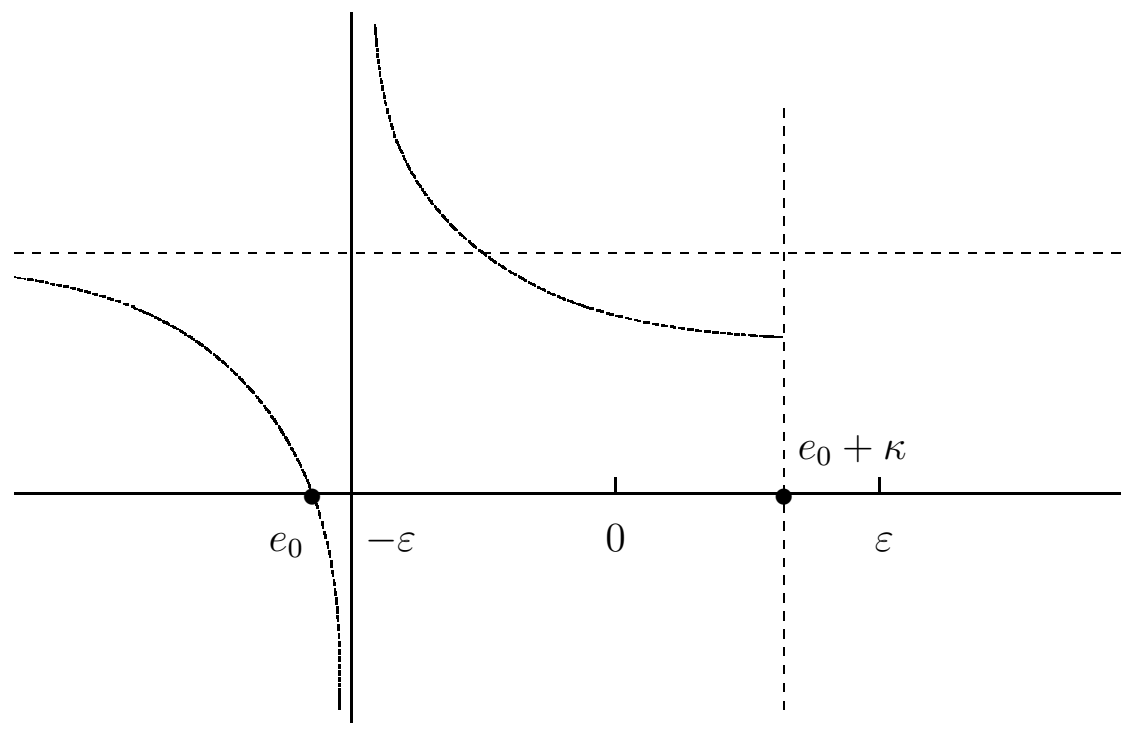




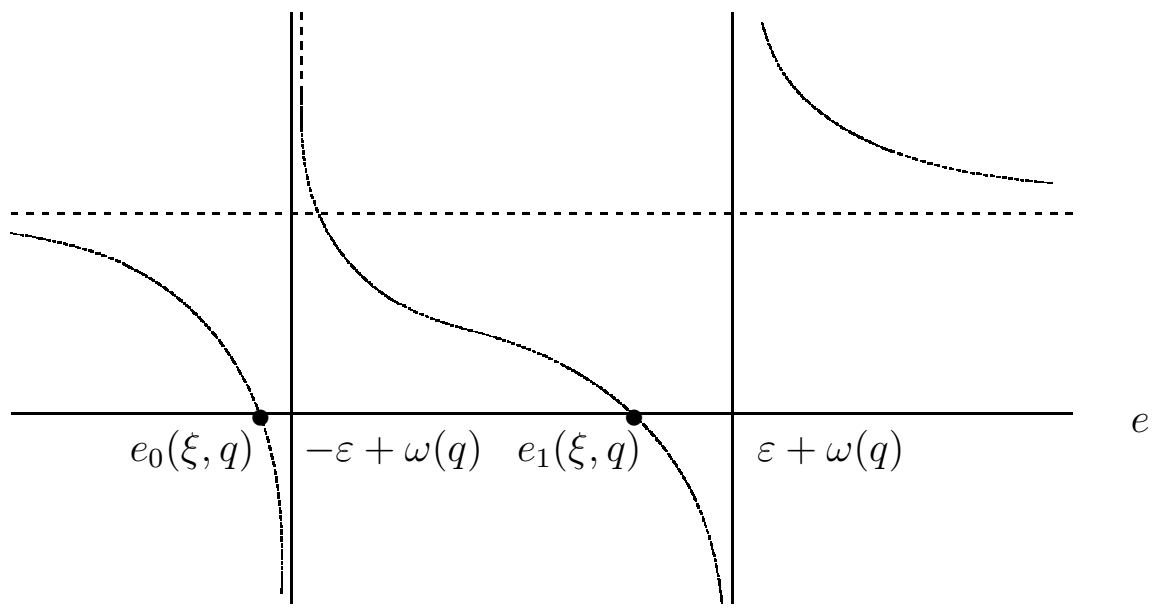




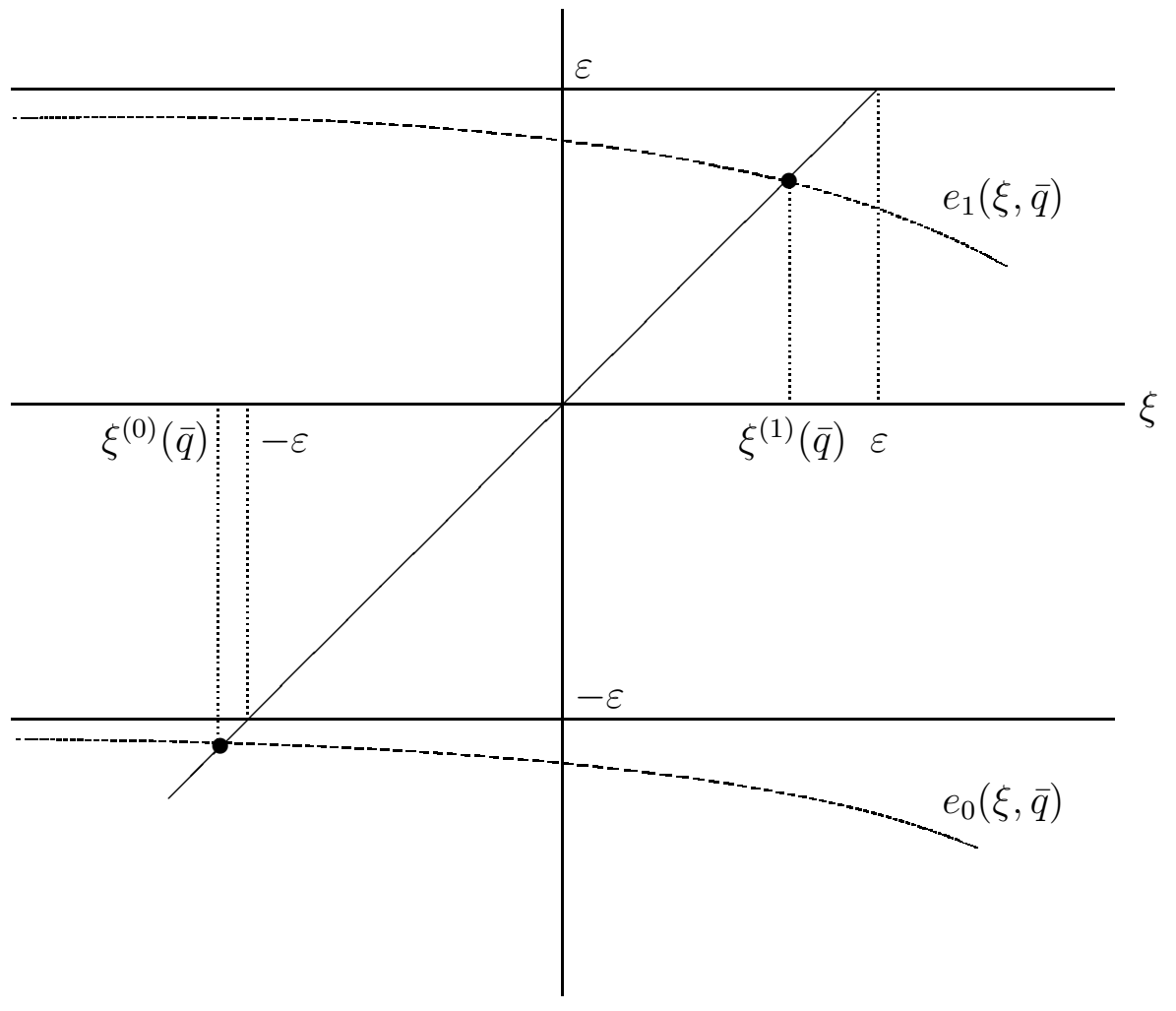

